\title{
HARMONIC TORI AND THEIR SPECTRAL DATA.
}

\author{
IAN MCINTOSH
}

One of the earliest applications of modern integrable systems theory (or "soliton theory") to differential geometry was the solution of the problem of finding all constant mean curvature (CMC) tori in $\mathbb{R}^{3}$ (and therefore, by taking the Gauss map, finding all non-conformal harmonic maps from a torus to $S^{2}$ ). At its simplest level this proceeds from the recognition that the Gauss-Codazzi equations of a CMC torus are the elliptic sinh-Gordon equations

$$
u_{z \bar{z}}+\sinh (4 u)=0, z=x+i y .
$$

It was shown in the late 1980's (24, 1] ) that each doubly periodic solution of this equation can be written down in terms of the Riemann $\theta$-function for a compact Riemann surface $X$, called the spectral curve (this also follows from Hitchin's work 10 on harmonic tori in $S^{3}$, which used a distinctly different approach). That this is true relies on two observations. First, (11) has a zero-curvature (or Lax pair) representation: it is the condition that

$$
\left[\frac{\partial}{\partial z}-U_{\zeta}, \frac{\partial}{\partial \bar{z}}+U_{\bar{\zeta}^{-1}}^{\dagger}\right]=0, U_{\zeta}=\left(\begin{array}{cc}
u_{z} & e^{-2 u} \zeta^{-1} \\
e^{2 u} \zeta^{-1} & -u_{z}
\end{array}\right), \forall \zeta \in \mathbb{C}^{*},
$$

where ' $\dagger$ ' denotes the Hermitian transpose. As a result this equation belongs a hierarchy of infinitely many commuting equations, so that solutions to (11) may belong to an infinite dimensional family of deformations through solutions. These deformations are called the "higher flows" of the sinhGordon hierarchy. Secondly, each independent higher flow contributes to the number of independent Jacobi fields which the CMC surface admits: these belong to the kernel of the elliptic operator $\triangle+4 \cosh (4 u)$. Thus for a torus there can only be finitely many independent higher flows. It follows that there must be a higher flow with respect to which the solution $u(z, \bar{z})$ is stationary. In this context this means there is a solution to

$$
d \xi_{\zeta}=\left[\xi_{\zeta}, \alpha_{\zeta}\right], \alpha_{\zeta}=U_{\zeta} d z-U_{\bar{\zeta}^{-1}}^{\dagger} d \bar{z}
$$

in which the matrix $\xi_{\zeta}(z, \bar{z})$ is a Laurent polynomial in $\zeta$ : it is called a polynomial Killing field. The spectral data of the CMC torus consists of the eigenvalues and eigenlines of $\xi_{\zeta}$. In particular, equation (2) means $\xi_{\zeta}$ is isospectral i.e. its characteristic polynomial is independent of $z$. This provides us a with an invariant planar algebraic curve which is essentially the Riemann surface $X$. Altogether the spectral data consists of the Riemann

Partially supported by EPSRC Grant GR/M97534. 
surface $X$, which always possesses a real involution, a rational function $\lambda$ on $X$ of degree 2 , and a line bundle $\mathcal{L}$ over $X$ satisfying a certain reality condition. The CMC surface is determined, up to Euclidean motions, by its spectral data. However, the existence of a polynomial Killing field is only a necessary condition for a CMC plane to be doubly periodic. If we call CMC planes "of finite type" when they possess a polynomial Killing field then one must still work at distinguishing the tori amongst the planes of finite type: this is a problem of closing periods on the surface (see e.g. [1, 8, 11]). This is also true for the Gauss map: the space of non-conformal harmonic maps $\varphi: \mathbb{R}^{2} \rightarrow S^{2}$ of finite type is substantially larger than the set of non-conformal harmonic tori.

Essentially the same line of argument shows that all non-isotropic harmonic tori in $\mathbb{C P}^{n}, S^{n}$ [4, 9] and all non-conformal harmonic tori in rank 1 compact symmetric spaces [5] are of (semisimple) finite type. Although the construction of the spectral data is more complicated the principle is the same [17, 18. However, these complications have the effect of obscuring the geometry of the original map. In 20 I proposed a more direct geometric construction of the map from the spectral data, and showed how this produces pluri-harmonic maps $\mathbb{R}^{2 k} \rightarrow G r_{k}\left(\mathbb{C}^{n+1}\right)$ as well.

My aim here is to use the example of non-conformal harmonic maps $\varphi: \mathbb{R}^{2} \rightarrow S^{2}$ as a way of motivating the geometric construction of [20]. To this end sections 1.1-1.5 describe the construction and properties of the spectral data for a map of semisimple finite type into $S^{2}$. The approach is more concrete than that of [17] and owes much to [9, 22, 25]. Having obtained the spectral data we examine it closely, in sections 1.6 and 1.7, to see exactly what is needed to reproduce the map. In particular, we obtain a clear understanding of the periodicity conditions by introducing a singularisation $X^{\prime}$ of $X$. Section 1.8 ties the previous discussion in with two other methods of reconstruction: the Symes' formula of [6] and the dressing orbit of the vacuum solution [7. I give explicit formulas for computing $\varphi$ from its (hyperelliptic) spectral curve. This is illustrated with the example of the bubbletons: these are CMC surfaces in $\mathbb{R}^{3}$ whose Gauss maps have rational nodal spectral curve. They are the solitons of CMC theory, some of which were known to geometers of the 19th century (see 21). The calculations in section 1.8 are particularly satisfying because they allow us to compute (using Nick Schmitt's CMCLab) explicit pictures of some CMC surfaces (see figures 1 and 2).

Section 2 describes the generalization presented in [20], which constructs pluri-harmonic maps of $\mathbb{R}^{2 k}$ into $G r_{k}\left(\mathbb{C}^{n+1}\right)$. The key point is that a pluriharmonic map $\varphi: \mathbb{R}^{2 k} \rightarrow G r_{k}\left(\mathbb{C}^{n+1}\right)$ of semisimple finite type arises as a composition: $\varphi=\psi \circ \gamma$ where

$$
\mathbb{R}^{2 k} \stackrel{\gamma}{\rightarrow} J\left(X^{\prime}\right) \stackrel{\psi}{\rightarrow} G r_{k}\left(\mathbb{C}^{n+1}\right) .
$$

The middle factor is the generalized Jacobian of a singularisation $X^{\prime}$ of the spectral curve $X$. The map $\gamma$ is a homomorphism and the map $\psi$ is algebraic, 
derived from a section of a trivial $G r_{k}\left(\mathbb{C}^{n+1}\right)$-bundle over $J\left(X^{\prime}\right)$. No proofs are given here, they can be found in [20]. Nevertheless, I give the details for the construction of totally equivariant maps, which are characterized as being those whose spectral curve is the Riemann sphere.

Acknowledgments. I am very grateful to Professors M Guest, R Miyaoka and Y Ohnita for their generous invitation to participate in the 9th MSJIRI in Tokyo, which was the motivation for this article. I am also grateful to Martin Kilian and Nick Schmitt for their ideas concerning the explicit construction of bubbletons, which motivated the discussion in section 1.8.

Notation. If $V$ is a vector space then $V^{t}$ will denote its dual, while $V^{*}$ will denote $V-\{0\}$.

\section{MAPS INTO $S^{2}$.}

1.1. Maps of semisimple finite type. Let us start with a harmonic map $\varphi: \mathbb{R}^{2} \rightarrow S^{2}$ of semisimple finite type. To recall what this means we fix a framing $F: \mathbb{R}^{2} \rightarrow S U_{2}$ with $F(0)=I$ i.e. $\varphi=F \cdot T$ if we view $S^{2} \simeq S U_{2} / T$ where $T$ is the maximal torus of diagonal matrices. The Lie algebra $\mathfrak{s u}_{2}$ splits into the vector space sum $\mathfrak{t}+\mathfrak{m}$ where $\mathfrak{t}$ contains all diagonal matrices and $\mathfrak{m}$ contains all off-diagonal matrices. Now define the $\mathfrak{s u}_{2}$-valued 1 -form $\alpha=F^{-1} d F$ : with respect to the splitting of $\mathfrak{s u}_{2}$ this decomposes into $\alpha_{\mathfrak{t}}+\alpha_{\mathfrak{m}}$. From these components we construct a $\mathbb{C}^{*}$-family of $\mathfrak{g l}_{2}$ - valued 1 -forms

$$
\alpha_{\zeta}=\zeta^{-1} \alpha_{\mathfrak{m}}^{(1,0)}+\alpha_{\mathfrak{t}}+\zeta \alpha_{\mathfrak{m}}^{(0,1)}
$$

where $\zeta \in \mathbb{C}^{*}$. The condition that $\varphi$ is harmonic is precisely the condition that $\alpha_{\zeta}$ satisfies the Maurer-Cartan equations for all $\zeta$. In addition, it has two symmetries:

$$
\alpha_{\bar{\zeta}^{-1}}=-\alpha_{\zeta}^{\dagger}, \quad \alpha_{-\zeta}=\nu\left(\alpha_{\zeta}\right)
$$

where ' $\nmid$ ' denotes the Hermitian transpose and for $A \in \mathfrak{g l}_{2}, \nu(A)=\operatorname{Ad} \tau \cdot A$ where

$$
\tau=\left(\begin{array}{cc}
1 & 0 \\
0 & -1
\end{array}\right)
$$

We say that $\varphi$ is of semisimple finite type when:

1a: there exists a smooth function $a: \mathbb{R}^{2} \rightarrow \mathbb{C}^{*}$ and a complex coordinate $z$ on $\mathbb{R}^{2}$ such that

$$
\alpha_{\mathfrak{m}}(\partial / \partial z)=\operatorname{Ad}\left(\begin{array}{cc}
a & 0 \\
0 & a^{-1}
\end{array}\right) \cdot\left(\begin{array}{cc}
0 & 1 \\
1 & 0
\end{array}\right)
$$

1b: there exists a smooth map $\xi_{\zeta}: \mathbb{R}^{2} \rightarrow C^{\omega}\left(\mathbb{C}^{*}, \mathfrak{g l}_{2}\right)$ satisfying

(i) $d \xi_{\zeta}+\left[\alpha_{\zeta}, \xi_{\zeta}\right]=0$

(ii) $\xi_{\zeta}$ also possesses the two symmetries in (3),

(iii) for all $z \in \mathbb{R}^{2}$ there is a positive integer $p$ such that $\xi_{\zeta}$ is a Laurent polynomial in $\zeta$ of order $2 p+1$. 
These properties together imply

$$
\xi_{\zeta}=\zeta^{-2 p-1} \alpha_{\mathfrak{m}}\left(\frac{\partial}{\partial z}\right)+\ldots+\zeta^{2 p+1} \alpha_{\mathfrak{m}}\left(\frac{\partial}{\partial \bar{z}}\right) .
$$

1.2. The symmetric spectral curve $\Sigma$. Define, for each $z \in \mathbb{R}^{2}$,

$$
\Sigma_{A}(z)=\left\{(\zeta,[v]) \in \mathbb{C}^{*} \times \mathbb{P}^{1}: \xi_{\zeta}(z) v=\mu v \exists \mu \in \mathbb{C}\right\} .
$$

To maximise the domain of definition here, whenever $\xi_{\zeta}$ is either singular or zero at $\zeta_{0}$ we replace it by $\left(\zeta-\zeta_{0}\right)^{m} \xi_{\zeta}$ where $m$ is chosen so that this is regular and non-zero at $\zeta_{0}$. It is clear that this describes an algebraic curve birationally equivalent to the planar curve with equation $\mu^{2}+\operatorname{det}\left(\xi_{\zeta}\right)=0$. Moreover $\Sigma_{A}(z)$ will be smooth (and unramified over the $\zeta$-plane) at all points for which $\xi_{\zeta}$ (or its renormalisation) is not nilpotent. In particular this is true over the unit $\zeta$-circle (for the symmetry conditions imply $\xi$ is skewHermitian there). Further, from (44) and 1a we see that $\Sigma_{A}(z)$ completes to a curve $\Sigma(z)$ in $\mathbb{P}^{1} \times \mathbb{P}^{1}$ by adding two smooth points over each of $\zeta=0$ and $\zeta=\infty$.

This curve admits a fixed point free involution arising from one of the symmetries of $\xi_{\zeta}$. Define

$$
\begin{aligned}
\tilde{\nu}: \mathbb{P}^{1} \times \mathbb{P}^{1} & \rightarrow \mathbb{P}^{1} \times \mathbb{P}^{1} \\
(\zeta,[v]) & \mapsto(-\zeta,[\tau v])
\end{aligned}
$$

Then $\tilde{\nu}$ induces a fixed point free involution on $\Sigma(z)$ and the quotient curve $\Sigma(z) / \tilde{\nu}$ is smooth wherever $\Sigma(z)$ is.

1.3. The quotient spectral curve $X$. Here we construct a model of the quotient curve $\Sigma(z) / \tilde{\nu}$. First, for any $\eta_{\zeta} \in C^{\omega}\left(\mathbb{C}^{*}, \mathfrak{g l}_{2}\right)$ satisfying $\nu\left(\eta_{\zeta}\right)=\eta_{-\zeta}$ define

$$
\hat{\eta}=\mathrm{Ad} \kappa \cdot \eta_{\zeta}, \quad \kappa=\left(\begin{array}{ll}
1 & 0 \\
0 & \zeta
\end{array}\right) .
$$

It is easy to check that $\hat{\eta}(-\zeta)=\hat{\eta}(\zeta)$ so that it is a function of $\lambda=\zeta^{2}$. Therefore, with an abuse of notation, let us use the notation

$$
\eta_{\lambda}=\mathrm{Ad} \kappa \cdot \eta_{\zeta}, \quad \lambda=\zeta^{2} .
$$

Now define

$$
X_{A}(z)=\left\{(\lambda,[w]) \in \mathbb{C}^{*} \times \mathbb{P}^{1}: \xi_{\lambda} w=\mu w\right\}
$$

with the same convention at singular points or zeroes of $\xi_{\lambda}$ as earlier. An easy computation shows that

$$
\xi_{\lambda}=\lambda^{-p-1}\left(\begin{array}{cc}
0 & a^{2} \\
0 & 0
\end{array}\right)+\ldots+\lambda^{p+1}\left(\begin{array}{cc}
0 & 0 \\
-\bar{a}^{2} & 0
\end{array}\right) .
$$

Therefore $X_{A}(z)$ is completed in $\mathbb{P}^{1} \times \mathbb{P}^{1}$ by adding the points $P_{0}=(0,[1,0])$ and $P_{\infty}=(\infty,[0,1])$. We will call this complete curve $X(z)$.

Lemma 1. $X(z)$ is isomorphic to the quotient curve $\Sigma(z) / \tilde{\nu}$. 
Proof. Let $f: \mathbb{C}^{*} \times \mathbb{P}^{1} \rightarrow \mathbb{C}^{*} \times \mathbb{P}^{1}$ be given by $f(\zeta,[v])=\left(\zeta^{2},[\kappa v]\right)$. Since $\xi_{\lambda}=\operatorname{Ad} \kappa \cdot \xi_{\zeta}$ this maps $\Sigma_{A}(z)$ onto $X_{A}(z)$ and exhibits it as an unramified double cover. Further, it is easy to check that $f \circ \tilde{\nu}=f$ so that $\Sigma_{A} / \tilde{\nu} \simeq X_{A}$. Finally, one readily checks that the restriction of $f$ to $\Sigma_{A}$ extends to $\Sigma$ with image $X$.

We deduce from this that $X(z)$ is smooth at both $P_{0}$ and $P_{\infty}$.

Lemma 2. $X(0) \simeq X(z)$ for all $z \in \mathbb{R}^{2}$.

Proof. By $1 \mathrm{~b}$ we have $d\left(\operatorname{Ad} F_{\lambda} \cdot \xi_{\lambda}(z)\right)=0$, where $F_{\lambda}$ is given by $F_{\lambda}^{-1} d F_{\lambda}=\alpha_{\lambda}$ and $F_{\lambda}(0)=I$. Hence

$$
\operatorname{Ad} F_{\lambda} \cdot \xi_{\lambda}(z)=\xi_{\lambda}(0)
$$

It follows that the map

$$
X_{A}(0) \rightarrow X_{A}(z) ; \quad(\lambda,[v]) \mapsto\left(\lambda,\left[F_{\lambda}^{-1} v\right]\right)
$$

is an isomorphism. To see that this extends to the complete curves we follow 9.

Define

$$
H_{+}=\exp \left(-z \lambda^{p} \xi_{\lambda}(0)\right) F_{\lambda} ; \quad H_{-}=\exp \left(-\bar{z} \lambda^{-p} \xi_{\lambda}(0)\right) F_{\lambda} .
$$

Then

$$
\begin{aligned}
H_{+}^{-1} d H_{+} & =-\operatorname{Ad} F_{\lambda}^{-1} \cdot \lambda^{p} \xi_{\lambda}(0) d z+\alpha_{\lambda} \\
& =-\lambda^{p} \xi_{\lambda}(z)+\alpha_{\lambda}
\end{aligned}
$$

which is polynomial in $\lambda$. Therefore $H_{+}$is holomorphic in $\lambda$. A similar computation shows that $H_{-}$is holomorphic in $\lambda^{-1}$. Whenever $\xi_{\lambda}(0) v=\mu v$ we see that

$$
F_{\lambda}^{-1} v=H_{+}^{-1} \exp \left(z \lambda^{p} \xi_{\lambda}(0)\right) v=e^{z \lambda^{p} \mu} H_{+}^{-1} v
$$

so that the line $\left[F_{\lambda}^{-1} v\right]$ equals $\left[H_{+}^{-1} v\right]$. Similarly we can show that $\left[F_{\lambda}^{-1} v\right]=$ $\left[H_{-}^{-1} v\right]$. Now, we also have

$$
\xi_{\lambda}(z)=\operatorname{Ad} F_{\lambda}^{-1} \cdot \xi_{\lambda}(0)=\operatorname{Ad} H_{ \pm}^{-1} \cdot \xi_{\lambda}(0)
$$

and it follows that the isomorphism (17) extends to give $X(0) \simeq X(z)$.

Remark. Notice that this proof shows that $\left.H_{+}\right|_{\lambda=0}$ is upper triangular since the isomorphism fixes the point $(0,[1,0])$. Likewise, $\left.H_{-}\right|_{\lambda=0}$ must be lower triangular.

1.4. The eigenline bundle $\mathcal{E}$ and its dual $\mathcal{L}$. Let $\mathcal{E}_{z}$ denote the eigenline bundle of $\xi_{\lambda}(z)$ : it is the pullback to $X(z)$ of the tautological bundle over $\mathbb{P}^{1}$, using the projection $(\lambda,[v]) \mapsto[v]$. We will denote its restriction to $X_{A}$ by $\mathcal{E}_{z, A}$ and, to avoid too many superscripts, we will denote the dual bundle by $\mathcal{L}_{z}$. The inclusion $\mathcal{E}_{z} \hookrightarrow \mathbb{P}^{1} \times \mathbb{P}^{1} \times \mathbb{C}^{2}$ pulls back the canonical coordinates $e_{1}, e_{2}$ on $\mathbb{C}^{2}$ to give two independent globally holomorphic sections of $\mathcal{L}_{z}$, which we also denote $e_{1}, e_{2}$ (or $e_{1}^{z}, e_{2}^{z}$ when necessary). Notice that $e_{1}\left((0,1)^{t}\right)=0$ and $e_{2}\left((1,0)^{t}\right)=0$ from which it follows that $e_{1} \in \Gamma\left(\mathcal{L}\left(-P_{\infty}\right)\right)$ and $e_{2} \in \Gamma\left(\mathcal{L}\left(-P_{0}\right)\right)$. 
Our next aim is to show that these sections span the space of global holomorphic sections of $\mathcal{L}$ and this characterizes $\mathcal{L}$. Indeed, one reason for working over $X$ is that on $\Sigma$ we do not have such a straightforward relationship between points and the sections arising from the coordinates $e_{1}, e_{2}$. First let $A=\mathbb{C}\left[X_{A}\right]$, the coordinate ring of $X_{A}$, let $B=\mathbb{C}\left[\lambda, \lambda^{-1}\right] \subset$ $A$ and for any ring $R$ use $R\langle\cdot\rangle$ to denote an $R$-module presented by its generators.

Theorem 1. (i) $\Gamma\left(\mathcal{L}_{A}\right)=B\left\langle e_{1}, e_{2}\right\rangle$, (ii) $\Gamma(\mathcal{L})=\mathbb{C}\left\langle e_{1}, e_{2}\right\rangle$, (iii) $\operatorname{deg} \mathcal{L}=g+1$ (where $g$ is the genus of $X$ ).

Proof. Let $Y$ be the completion of the planar curve with equation $\mu^{2}+$ $\operatorname{det}\left(\xi_{\lambda}\right)=0$, with $Y_{A}$ that part of the curve lying over $\lambda \neq 0, \infty$. Then $Y_{A}$ has coordinate ring $A_{Y}=\mathbb{C}\left[\lambda, \lambda^{-1}, \mu\right] \subset A$ and there is a degree 1 morphism $\alpha: X \rightarrow Y$. Set $M=\Gamma\left(X_{A}, \mathcal{L}\right)=\Gamma\left(Y_{A}, \alpha_{*} \mathcal{L}\right)$. First we will show that $B\left\langle e_{1}, e_{2}\right\rangle \subset M$ is an $A_{Y}$-submodule. For any $v \in \Gamma\left(Y_{A}, \alpha_{*} \mathcal{E}\right)=\Gamma\left(X_{A}, \mathcal{E}\right)$ we have $v=\left(e_{1}(v), e_{2}(v)\right)^{t}$ and $\xi_{\lambda} v=\mu v$ implies $\mu e_{i}(v)=a_{i} e_{1}(v)+b_{i} e_{2}(v)$ for some $a_{i}, b_{i} \in B$. It follows that $\mu e_{i} \in B\left\langle e_{1}, e_{2}\right\rangle$. So $B\left\langle e_{1}, e_{2}\right\rangle$ is an $A_{Y}$-module.

Now it can only be a proper $A_{Y}$-module if its localisation at every maximal ideal $\mathfrak{p}$ is also proper in the corresponding localisation $M_{\mathfrak{p}}$. But at any smooth point $P \in Y_{A}$, with maximal ideal $\mathfrak{p}, M_{\mathfrak{p}}$ is the stalk $\mathcal{L}_{P}$ of $\mathcal{L}$ at $P$, and all its proper submodules are contained in $\mathcal{L}_{P}(-P)$ (the local sections which vanish at $P$ ). But in that case every section in $M$ must vanish at $P$. This means that for every $v \in \mathcal{E}_{P}$ both $e_{1}(v)$ and $e_{2}(v)$ vanish at $P$, which is absurd (there is always a non-zero eigenvector). Since $Y_{A}$ must have at least one smooth point we deduce that $M=B\left\langle e_{1}, e_{1}\right\rangle$.

(ii) Given (i) it suffices to show that if $\lambda^{n} e_{i} \in \Gamma(\mathcal{L})$ for $n \in \mathbb{Z}$ then $n=0$. Since $e_{1}$ does not vanish at $P_{0}$ and $e_{2}$ does not vanish at $P_{\infty}$ it suffices to show that neither $\lambda e_{1}$ nor $\lambda^{-1} e_{2}$ are globally holomorphic. Consider first $\lambda e_{1}$ : it is globally holomorphic if and only if $\lambda e_{1}(v)$ is holomorphic at $P_{\infty}$ for all locally holomorphic sections $v$ of $\mathcal{E}$ about $P_{\infty}$. By definition,

$$
[v]=\left[\left(\begin{array}{cc}
1 & 0 \\
0 & \pm \zeta
\end{array}\right) w\right]
$$

where $\zeta^{-2 p-1} \xi_{\zeta} w=\mu \zeta^{-2 p-1} w$. We may assume, without loss of generality, that

Therefore

$$
w=\left(\begin{array}{c}
\bar{a}^{-1}+O\left(\zeta^{-1}\right) \\
\pm \bar{a}+O\left(\zeta^{-1}\right)
\end{array}\right)
$$

$$
v=\left(\begin{array}{c}
\zeta^{-1} \bar{a}^{-1}+O\left(\zeta^{-2}\right) \\
\bar{a}+O\left(\zeta^{-1}\right)
\end{array}\right)
$$

Hence $\lambda e_{1}(v)$ has a first order pole at $P_{\infty}$. A similar calculation shows for $v$ a locally holomorphic section about $P_{0}$ we can take

$$
v=\left(\begin{array}{c}
a+O(\zeta) \\
\zeta a^{-1}+O\left(\zeta^{2}\right)
\end{array}\right)
$$


and therefore $\lambda^{-1} e_{2}(v)$ has a first order pole at $P_{0}$.

(iii) Since $\lambda$ has divisor $2 P_{0}-2 P_{\infty}$, (i) and (ii) imply $\Gamma\left(\mathcal{L}\left(-2 P_{0}\right)\right)=0$. So applying the Riemann-Roch formula gives $\operatorname{deg} \mathcal{L} \leq g+1$. Now we show $\operatorname{deg} \mathcal{L} \geq g+1$. For $n$ any suitably large positive integer $\mathcal{L}\left(2 n P_{\infty}\right)$ must be non-special so that

$$
\operatorname{dim} \Gamma\left(\mathcal{L}\left(2 n P_{\infty}\right)\right)=\operatorname{deg} \mathcal{L}+2 n+1-g .
$$

But

$$
\mathbb{C}\left\langle e_{1}, e_{2}, \lambda e_{1}, \lambda e_{2}, \ldots, \lambda^{n} e_{1}, \lambda^{n} e_{2}\right\rangle \subset \Gamma\left(\mathcal{L}\left(2 n P_{\infty}\right)\right)
$$

so $\operatorname{deg} \mathcal{L}+2 n+1-g \geq 2 n+2$.

1.5. The real structure of $\Gamma(\mathcal{L})$. An important property of $\Gamma(\mathcal{L})$ is that possesses a Hermitian inner product: this comes from a reality condition on $\mathcal{L}$ and is essential since we intend to identify $\mathbb{P} \Gamma(\mathcal{L})$ with $S^{2} \cong \mathbb{C P}^{1}$ as a Hermitian symmetric space. This reality condition arises as follows.

The real symmetry $\xi_{\bar{\lambda}-1}=-\xi_{\lambda}^{\dagger}$ induces a real involution $\rho$ on $X$ for which $\overline{\rho_{*} \lambda}=\lambda^{-1}$ and $\overline{\rho_{*} \mu}=-\mu$. Notice that, since $\xi_{\lambda}$ is skew-Hermitian over $|\lambda|=1, \mu$ is pure imaginary there so $\rho$ fixes all points over $|\lambda|=1$ this will prove to be important later. Consequently the eigenline bundle $\mathcal{E}$ also satisfies a reality condition.

Proposition 1. $\overline{\rho_{*} \mathcal{E}} \simeq \mathcal{L}(-R)$ where $R$ is the ramification divisor of $\lambda$ : $X \rightarrow \mathbb{P}^{1}$.

Proof. Since $\overline{\rho_{*} \xi_{\lambda}}=-\xi_{\lambda}^{t}$ sections of $\overline{\rho_{*} \mathcal{E}}$ correspond to solutions of

$$
\xi_{\lambda}^{t} w=\mu w .
$$

Take any proper open subset $U \subset X$ for which $U=\lambda^{-1} \circ \lambda(U)$, and let $v: U \rightarrow \mathcal{E}$ be a trivialising section. If $\sigma$ denotes the hyperelliptic involution on $X$ then $\sigma_{*} \mu=-\mu$ and clearly $v, \sigma_{*} v$ are linearly independent at $P \in X$ if and only if $P$ is not a ramification point. Take $V$ to be the matrix with columns $v, \sigma_{*} v$, then we have $\operatorname{det}(V)$ vanishing only at ramification points. Define $W=\operatorname{det}(V) \cdot V^{-1 t}$, then $W$ is holomorphic in $U$ and

$$
\xi_{\lambda}^{t} W=W\left(\begin{array}{cc}
\mu & 0 \\
0 & -\mu
\end{array}\right) .
$$

It is easy to check that the columns of $W$ are given by $w,-\sigma_{*} w$ where $e_{1}(w)=e_{2}\left(\sigma_{*} v\right)$ and $e_{2}(w)=-e_{1}\left(\sigma_{*} v\right)$. Therefore $w$ corresponds to a trivialising section for $\overline{\rho_{*} \mathcal{E}}$ over $U$. Now consider the injective homomorphism of $\mathcal{O}_{U}$-modules

$$
\begin{aligned}
\mathcal{O}_{U}\langle w\rangle & \rightarrow \operatorname{Hom}\left(\mathcal{O}_{U}\langle v\rangle, \mathcal{O}_{U}\right) \\
f . w & \mapsto\left(h . v \mapsto f h . w^{t} v\right)
\end{aligned}
$$

for $f, h \in \mathcal{O}_{U}$. Since $w^{t} v=\operatorname{det}(V)$ we see that the induced sequence of sheaves is

$$
0 \rightarrow \overline{\rho_{*} \mathcal{E}} \rightarrow \mathcal{L} \rightarrow \mathcal{O}_{R} \rightarrow 0
$$

where $\mathcal{O}_{R}$ is the skyscraper sheaf for the divisor $R$. Therefore $\overline{\rho_{*} \mathcal{E}} \simeq \mathcal{L}(-R)$. 
Consequently we have $\mathcal{L} \otimes \overline{\rho_{*} \mathcal{L}} \simeq \mathcal{O}_{X}(R)$. The inner product on $\mathbb{C}^{2}$ corresponds (at least over the unit circle) to the section

$$
e_{1} \otimes \overline{\rho_{*} e_{1}}+e_{2} \otimes \overline{\rho_{*} e_{2}}
$$

(which maps $(v, w)$ to ${\overline{\rho_{*} v}}^{t} w$ ). Up to scaling this corresponds to an inner product on $\Gamma(\mathcal{L})$ determined in the following manner.

We first take any $s \in \Gamma(\mathcal{L})$ to identify $\mathcal{L}$ with the divisor line bundle $\mathcal{O}_{X}(D)$ where $D$ is the divisor of zeroes of $s$. Second we fix a rational function $f$ with divisor $D+\rho_{*} D-R$ for which $\overline{\rho_{*} f}=f$ and $f$ is positive over $|\lambda|=1$ (this is always possible since $\rho$ fixes all points over the unit circle). Now we define

$$
\begin{aligned}
& h: \Gamma(\mathcal{L}) \times \Gamma(\mathcal{L}) \rightarrow \mathbb{C} ; \\
& h\left(s_{1}, s_{2}\right)=\frac{1}{2} \sum_{j=1}^{2} f\left(O_{j}\right)\left(s_{1} / s\right)\left(O_{j}\right) \overline{\rho_{*}\left(s_{2} / s\right)}\left(O_{j}\right)
\end{aligned}
$$

where $O_{1}, O_{2}$ are the two points over $\lambda=1$. The proper interpretation of the right hand side is in terms of the trace map $\operatorname{Tr}: \mathcal{O}_{X}(R) \rightarrow \mathbb{C}$ which I will not explain in detail here (see, for example, 26]). But it is worth noting for future reference that this inner product clearly makes the subspaces $\Gamma\left(\mathcal{L}\left(-O_{1}\right)\right), \Gamma\left(\mathcal{L}\left(-O_{2}\right)\right) \subset \Gamma(\mathcal{L})$ orthogonal.

1.6. What the frame does. Let us introduce $\hat{\Gamma}(\cdot)$ for spaces of analytic sections and let $\hat{A}$ denote the ring of analytic functions on $X_{A}$ while $\hat{B}$ denotes the analytic functions on the punctured $\lambda$-plane $\mathbb{P}_{\lambda}^{1} \backslash\{0, \infty\}$. The map

$$
\hat{\Gamma}\left(\mathcal{E}_{z, A}\right) \rightarrow \hat{\Gamma}\left(\mathcal{E}_{0, A}\right) ; \quad v \mapsto F_{\lambda} v
$$

is clearly an isomorphism of $\hat{A}$-modules. Therefore it corresponds to a family of trivialising sections

$$
\theta_{z} \in \hat{\Gamma}\left(\mathcal{E}_{0, A} \otimes \mathcal{L}_{z, A}\right) .
$$

Let $J(X)$ denote the Jacobian of $X$ - the abelian variety of isomorphism classes of line bundles of degree zero. If $J_{R}(X)$ denotes the real subgroup of degree zero line bundles $L$ for which $\overline{\rho_{*} L} \simeq L^{-1}$ then we deduce from the previous section that $\mathcal{E}_{0} \otimes \mathcal{L}_{z}$ belongs to $J_{R}(X)$ for all $z$.

Proposition 2. (i) Define $L: \mathbb{R}^{2} \rightarrow J_{R}(X)$ by $L_{z}=\mathcal{E}_{0} \otimes \mathcal{L}_{z}$. Then $L$ is $\mathbb{R}$-linear (i.e. a homomorphism of real abelian groups). (ii) $\theta_{z} \exp \left(z \lambda^{p} \mu\right.$ ) is holomorphic and non-vanishing over $P_{0}$ while $\theta_{z} \exp \left(\bar{z} \lambda^{-p} \mu\right)$ is holomorphic and non-vanishing over $P_{\infty}$.

Proof. Observe that (ii) implies (i) since we deduce from it that $L_{z}$ corresponds to the transition functions $\exp \left(z \lambda^{p} \mu\right)$ and $\exp \left(\bar{z} \lambda^{-p} \mu\right)$ patching from $X_{A}$ to $U_{0}$ and $U_{\infty}$ respectively, where the latter are open neighbourhoods of $P_{0}$ and $P_{\infty}$ respectively.

To prove (ii) we recall from the proof of lemma 2 that if $v_{0}$ is a holomorphic section of $\mathcal{E}_{0}$ about $P_{0}$ then $F_{\lambda}^{-1} v_{0}=H_{+}^{-1} \exp \left(z \lambda^{p} \mu\right) v_{0}$ so that $\exp \left(-z \lambda^{p} \mu\right) F_{\lambda}^{-1} v_{0}$ is a holomorphic section of $\mathcal{E}_{z}$ about $P_{0}$. But $F_{\lambda}^{-1} v_{0}$ 
corresponds to $v_{0} \otimes \theta_{z}^{-1}$ so tensoring with $\exp \left(-z \lambda^{p} \mu\right) \theta_{z}^{-1}$ preserves holomorphicity about $P_{0}$. A similar argument using $H_{-}$about $P_{\infty}$ proves the second part of (ii).

Let $L_{z, A}$ denote the restriction to $X_{A}$ of $L_{z}$. We want to make explicit the representation

$$
\mathcal{F}: \hat{\Gamma}\left(L_{A}\right) \rightarrow \hat{B} \otimes \mathfrak{g l}_{2}
$$

which gives us $\mathcal{F}(\theta)=F_{\lambda}$. It arises from the composite isomorphism

$$
\epsilon_{z}: \hat{\Gamma}\left(\mathcal{E}_{z, A}(R)\right) \rightarrow \hat{B} \otimes \Gamma\left(\mathcal{L}_{z}\right)^{t} \rightarrow \hat{B} \otimes \mathbb{C}^{2} .
$$

The second arrow is just the identification $\Gamma\left(\mathcal{L}_{z}\right)^{t} \rightarrow \mathbb{C}^{2}$ determined by $e_{1}^{z}, e_{2}^{z}$. The first arrow is the $\hat{B}$-module isomorphism dual to $\hat{B} \otimes \Gamma(\mathcal{L}) \simeq \hat{\Gamma}\left(\mathcal{L}_{A}\right)$, from theorem 1. This uses the fact, implicit in the proof of proposition 1 that $\lambda_{*} \mathcal{L}$ is dual to $\lambda_{*} \mathcal{E}(R)$. It follows that to any $\phi \in \hat{\Gamma}\left(L_{A}\right)$ there is some $\mathcal{F}(\phi) \in \hat{B} \otimes \mathfrak{g l}_{2}$ so that the following diagram commutes:

$$
\begin{array}{ccc}
\hat{\Gamma}\left(\mathcal{E}_{z, A}(R)\right) & \stackrel{\phi}{\rightarrow} & \hat{\Gamma}\left(\mathcal{E}_{0, A}(R)\right) \\
\epsilon_{z} \downarrow & & \downarrow \epsilon_{0} \\
\hat{B} \otimes \mathbb{C}^{2} & \stackrel{\mathcal{F}(\phi)}{\rightarrow} & \hat{B} \otimes \mathbb{C}^{2} .
\end{array}
$$

Next we will show that $\theta$ is almost completely determined by it's behaviour at the points $P_{0}, P_{\infty}$. First observe that from (41) we have

$$
\mu^{2}=-\operatorname{det} \xi_{\zeta}=\zeta^{-4 p-2}+\ldots+\zeta^{4 p+2}=\lambda^{-2 p-1}+\ldots+\lambda^{2 p+1} .
$$

Since $\overline{\rho_{*} \mu}=-\mu$ whereas $\overline{\rho_{*} \zeta}=\zeta^{-1}$ we find, with the right sign choice for $\zeta, \mu=\zeta^{-2 p-1}+\ldots-\zeta^{2 p+1}$. Therefore $\lambda^{p} \mu-\zeta^{-1}$ is holomorphic about $P_{0}$ while $\lambda^{-p} \mu+\zeta$ is holomorphic about $P_{\infty}$. Consequently, as a corollary of proposition 2 we have:

Corollary 1. $\theta_{z}$ is determined up to sign, amongst trivialising sections of $L_{A}$, by the properties that: (a) $\theta_{z} \exp \left(z \zeta^{-1}\right)$ is holomorphic and nonvanishing over $P_{0}$ while $\theta_{z} \exp (-\bar{z} \zeta)$ is holomorphic and non-vanishing over $P_{\infty}$ and, (b) $\operatorname{det}\left(\mathcal{F}\left(\theta_{z}\right)\right)=1$.

Proof. If $\phi$ is any other trivialising section with these properties then $\phi \theta_{z}^{-1}$ is a globally holomorphic function and therefore a constant, $k$ say. But clearly $\operatorname{det}\left(\mathcal{F}\left(k \theta_{z}\right)\right)=k^{2}$ so that $k= \pm 1$.

Remark. The unitary nature of $\mathcal{F}\left(\theta_{z}\right)$ on the unit circle is a reflection of the fact that $\overline{\rho_{*} \theta}=\theta^{-1}$.

Finally, let us use this corollary to display a simple characterisation for the map $L$. Since it is linear it is completely determined by $d L_{0}(\partial / \partial z)$ which lies in $T_{1}^{1,0} J(X)$ (here 1 denotes the identity in $J(X)$ ). By the corollary above $L(z)$ corresponds to the cohomology class $[c(z)]$ in $H^{1}\left(X, \mathcal{O}^{*}\right)$ for the 1-cocycle

$$
c(z)=\left\{\left(e^{z \zeta^{-1}}, X_{A}, U_{0}\right),\left(e^{-\bar{z} \zeta}, X_{A}, U_{\infty}\right)\right\}
$$


for the open cover $X_{A}, U_{0}, U_{\infty}$, where now $U_{0}, U_{\infty}$ are (disjoint) parameter discs (i.e. domains for $\zeta, \zeta^{-1}$ ). Therefore

$$
\left.\frac{\partial[c]}{\partial z}\right|_{z=0}=\left[\left(\zeta^{-1}, X_{A}, U_{0}\right),\left(1, X_{A}, U_{\infty}\right)\right] \in H^{1}(X, \mathcal{O}) .
$$

Now recall the isomorphism $H^{1}(X, \mathcal{O}) \simeq \Gamma\left(\Omega_{X}\right)^{t}$ : it identifies $\partial[c] / \partial z$ with the map $f: \omega \mapsto \operatorname{res}_{P_{0}} \zeta^{-1} \omega$ for $\omega \in \Gamma\left(\Omega_{X}\right)$. But now observe that

$$
\operatorname{res}_{P_{0}} \zeta^{-1} \omega=(\omega / d \zeta)\left(P_{0}\right)=\frac{\partial}{\partial \zeta} \int_{P_{0}}^{\zeta} \omega .
$$

Hence $f=d \mathcal{A}_{P_{0}}(\partial / \partial \zeta)$ where $\mathcal{A}_{P_{0}}: X \rightarrow J(X)$ is the Abel map with base point $P_{0}$. Thus we learn:

Lemma 3. The linear map $L: \mathbb{R}^{2} \rightarrow J_{R}(X)$ is uniquely determined by the property that $d L_{0}(\partial / \partial z)=d \mathcal{A}_{P_{0}}(\partial / \partial \zeta)$.

1.7. Periodicity conditions. We have seen that the non-conformal doubly periodic harmonic map $\varphi: \mathbb{R}^{2} \rightarrow S^{2}$ yields us spectral data $(X, \lambda, \mathcal{L})$ and it is easy to see how to reverse this procedure to reconstruct the map from this data. We first construct the linear map $L: \mathbb{R}^{2} \rightarrow J_{R}(X)$ given by lemma 3 and define $\mathcal{L}_{z}=\mathcal{L} \otimes L_{z}$. By theorem $1 \Gamma\left(\mathcal{L}_{z}\right)$ comes equipped with a frame $e_{1}^{z}, e_{2}^{z}$ determined by the points $P_{\infty}, P_{0}$ : this frame is chosen to be unitary according to the trace inner product described above. With the frame we recover the map $\mathcal{F}$ in (11). Now we equip $L_{z}$ with the unique (up to sign) trivialising section $\theta_{z}$ over $X_{A}$ given by corollary 1. Thus we obtain the extended frame $F_{\lambda}=\mathcal{F}\left(\theta_{z}\right)$ and the map $\varphi$ is recovered as $F_{1} \circ[1,0]$ where $[1,0] \in \mathbb{C P}^{1}$.

However, we do not need the frame itself to obtain $\varphi$ : it is clear that each line $\varphi(z) \in \mathbb{C P}^{1}$ corresponds to the line $\Gamma\left(\mathcal{L}_{z}\left(-P_{\infty}\right)\right) \in \mathbb{P} \Gamma\left(\mathcal{L}_{z}\right)$ where $\mathbb{P} \Gamma\left(\mathcal{L}_{z}\right)$ is identified with $\mathbb{C P}^{1}$ using $\theta_{z}$. There is an invariant way of describing this identification which avoids explicit reference to $\theta_{z}$ and this helps us understand the periodicity conditions. To obtain this let us first consider the expression for $\varphi$ in homogeneous coordinates: it can be written as

$$
\varphi=\left[\left.\left(e_{1}^{0} f_{1}^{z} \theta_{z}\right)\right|_{\lambda=1},\left.\left(e_{2}^{0} f_{1}^{z} \theta_{z}\right)\right|_{\lambda=1}\right],
$$

where $f_{j}^{z} \in \Gamma\left(\mathcal{E}_{z, A}(R)\right)$ is the $B$-module generator dual to to $e_{j}^{z}$. Now it is clear that if we choose some other (unitary) basis $v_{1}, v_{2}$ of $\Gamma(\mathcal{L})$ we obtain, up to isometry of $S^{2}$, the same map. In particular, following the remarks made earlier, we could choose this new basis such that $v_{1}$ vanishes at $O_{2}$ and $v_{2}$ vanishes at $O_{1}$. In that case

$$
\varphi=\left[\left.\left(f_{1}^{z} \theta_{z}\right)\right|_{O_{1}},\left.\alpha\left(f_{1}^{z} \theta_{z}\right)\right|_{O_{2}}\right]
$$

where $\alpha: \mathcal{L}\left|O_{2} \rightarrow \mathcal{L}\right| O_{1}$ is the fibre identification induced by the choice of $v_{1}, v_{2}$ (i.e. $\left.\alpha\left(\left.v_{2}\right|_{O_{2}}\right)=\left.v_{1}\right|_{O_{1}}\right)$.

Recall that up to scaling $f_{1}$ is determined purely by the vanishing of $e_{1}$ at $P_{\infty}$. It follows that $\varphi$ has periodicity $\varphi(z+\tau)=\varphi(z)$ precisely when both 
equations $L(z+\tau)=L(z)$ and

$$
\left.\left(\theta_{z+\tau} \theta_{z}^{-1}\right)\right|_{O_{2}}=\left.\left(\theta_{z+\tau} \theta_{z}^{-1}\right)\right|_{O_{1}}
$$

are satisfied. The latter condition is more simply interpreted as saying that the fibre identification $L_{z}\left|O_{2} \rightarrow L_{z}\right| O_{1}$ given by $\left.\left.\theta_{z}\right|_{O_{2}} \mapsto \theta_{z}\right|_{O_{1}}$ is $\tau$-periodic. This identication determines at each $z$ a line bundle $L_{z}^{\prime}$ over $X^{\prime}$, the singular curve obtained from $X$ by identifying $O_{1}$ with $O_{2}$ to obtain a node. Thus we have a $\tau$-periodic map $L^{\prime}$ from $\mathbb{R}^{2}$ to $J\left(X^{\prime}\right)$, the (generalized) Jacobi variety for $X^{\prime}$. Recall that the pullback of line bundles along $X \rightarrow X^{\prime}$ induces a surjective homomorphism $\pi: J\left(X^{\prime}\right) \rightarrow J(X)$ whose fibre at $L$ is $L\left|O_{2} \otimes L^{-1}\right| O_{1} \cong \mathbb{C}^{*}$. In fact $\overline{\rho_{*} L^{\prime}} \cong L^{\prime-1}$ so $L^{\prime}$ takes values in a real subgroup $J_{R}\left(X^{\prime}\right)$ of $J\left(X^{\prime}\right)$. It can be shown that, when $X$ is smooth of genus $g$, this group is a real compact torus of dimension $g+1$.

Lemma 4. The map $L^{\prime}: \mathbb{R}^{2} \rightarrow J_{R}\left(X^{\prime}\right)$ defined above is linear and is uniquely determined by the property that $d L_{0}^{\prime}(\partial / \partial z)=d \mathcal{A}_{P_{0}}^{\prime}(\partial / \partial \zeta)$, where $\mathcal{A}_{P_{0}}^{\prime}: X^{\prime}-\{O\} \rightarrow J\left(X^{\prime}\right)$ is the Abel map for $X^{\prime}$ based at $P_{0}$.

Proof. Since $\theta_{z}$ arises from the 1-cocycle $c(z)$ in (12) $L_{z}^{\prime}$ has 1-cocycle

$$
c^{\prime}(z)=\left\{\left(e^{z \zeta^{-1}}, X_{A}^{\prime}, U_{0}\right),\left(e^{-\bar{z} \zeta}, X_{A}^{\prime}, U_{\infty}\right)\right\} .
$$

Now recall (from e.g. 26]) that

$$
J\left(X^{\prime}\right) \simeq \Gamma\left(\Omega_{X}^{\prime}\right)^{t} / H_{1}\left(X-\left\{O_{1}, O_{2}\right\}, \mathbb{Z}\right),
$$

where $\Omega_{X}^{\prime}$ is the sheaf of regular differentials on $X^{\prime}$ : each such differential can be identified with a meromorphic differential on $X$ whose only poles are simple ones at $O_{1}$ and $O_{2}$ i.e. $\Omega_{X}^{\prime} \cong \Omega_{X}\left(O_{1}+O_{2}\right)$. The Abel map for $X^{\prime}$ is defined by

$$
\mathcal{A}_{P_{0}}^{\prime}: X^{\prime}-\{O\} \rightarrow J\left(X^{\prime}\right) ; \quad P \mapsto \int_{P_{0}}^{P},
$$

where $O$ is the nodal point lying under $O_{1}, O_{2}$. To compute $d L_{0}^{\prime}$ we simply repeat the computation prior to lemma 3 using $c^{\prime}(z)$.

Corollary 2. The harmonic map $\varphi: \mathbb{R}^{2} \rightarrow S^{2}$ with spectral data $X, \lambda, \mathcal{L}$ has period $\tau$ if and only if the related map $L^{\prime}: \mathbb{R}^{2} \rightarrow J_{R}\left(X^{\prime}\right)$ has period $\tau$. This depends only on the data $X, \lambda$.

In particular, if $X$ has genus $g \leq 1$ the harmonic map is necessarily doubly periodic since $J_{R}\left(X^{\prime}\right)$ is topologically $S^{1}$ or $S^{1} \times S^{1}$. These examples yield the Gauss maps of all Delaunay surfaces in $\mathbb{R}^{3}$ (i.e. the constant mean curvature surfaces of revolution) with the case $g=0$ corresponding to the Gauss map of the cylinder. A more interesting class of singly periodic examples are the Gauss maps of the "bubbletons". The bubbletons are periodic CMC surfaces whose ends are asymptotic to the standard cylinder (see figure 2 ). They get their name because they correspond to soliton solutions of the sinh-Gordon equation, which governs the behaviour of the metric. As with $\mathrm{KdV}$ solitons, these solutions have rational nodal spectral curves. Using the theory above we can characterize these spectral curves as follows. 
Proposition 3. Let $X$ be the rational nodal curve of arithmetic genus $g=$ $2 r$ with equation

$$
\mu^{2}=\lambda \prod_{j=1}^{r}\left(\lambda-a_{j}\right)^{2}\left(1-a_{j} \lambda\right)^{2}, a_{j} \in \mathbb{R}, 0<a_{j}<1 .
$$

Then $X, \lambda$ is the spectral data for a singly periodic non-conformal harmonic $\operatorname{map} \varphi: \mathbb{R}^{2} \rightarrow S^{2}$ if and only if there exist positive integers $p_{0}, p_{1}, \ldots, p_{r}$ for which

$$
a_{j}=\left(\frac{p_{j}}{p_{0}} \pm \sqrt{\frac{p_{j}^{2}}{p_{0}^{2}}-1}\right)^{2}, j=1, \ldots, r .
$$

Proof. Let us set $\zeta=\sqrt{\lambda}$ : this is a rational coordinate on $X$. Thus we identify $X$ with the singularization of the Riemann sphere $\mathbb{P}_{\zeta}$ with the points $\pm \zeta_{j}$ identified, where $\zeta_{j}^{2}=a_{j}$ and $\zeta_{r+j}^{2}=a_{j}^{-1}$ for $j=1, \ldots, r$. Notice that $\zeta_{j} \in \mathbb{R}$ since $a_{j}>0$. $X^{\prime}$ is the further singularization obtained by additionally identifying $\pm \zeta_{0}$, where $\zeta_{0}=1$. We may assume the real involution is $\rho_{*} \zeta=\bar{\zeta}^{-1}$. A basis for $\Gamma\left(\Omega_{X}^{\prime}\right)$ is given by

$$
\omega_{j}=\frac{1}{2 \pi i}\left(\frac{1}{\zeta-\zeta_{j}}-\frac{1}{\zeta+\zeta_{j}}\right) d \zeta, j=0, \ldots, 2 r,
$$

Now $X^{\prime}$ is obtained from its normalisation (a smooth curve of genus $2 r+1$ ) by shrinking half the homology generators to zero, hence $H_{1}\left(X^{\prime}, \mathbb{Z}\right)$ is generated by $\gamma_{j}, j=0, \ldots, 2 r$ where each of these is the boundary of a small positively oriented disc containing $\zeta_{j}$. It follows that $\oint_{\gamma_{j}} \omega_{k}=\delta_{j k}$. The real group $J_{R}\left(X^{\prime}\right)$ is isomorphic to

$$
\left\{\omega \in \Gamma\left(\Omega_{X}^{\prime}\right): \rho_{*} \omega=-\bar{\omega}\right\}^{t} /\left\{\gamma \in H_{1}\left(X^{\prime}, \mathbb{Z}\right): \rho_{*} \gamma \sim-\gamma\right\}
$$

which we will write more simply as $V^{t} / \Gamma$. It is not hard to check that a basis for $V$ is given by

$$
v_{0}=\omega_{0}, v_{j}=\frac{1}{2}\left(\omega_{j}+\omega_{r+j}\right), v_{r+j}=\frac{i}{2}\left(\omega_{j}-\omega_{r+j}\right), j=1, \ldots, r
$$

and generators for $\Gamma \subset V^{t}$ can be given by

$$
\oint_{\gamma_{0}}, \oint_{\gamma_{j}+\gamma_{r+j}}, j=1, \ldots, r \text {. }
$$

With respect to this basis for $V$ the dual isomorphism $V^{t} \cong \mathbb{R}^{2 r+1}$ identifies the generators for $\Gamma$ with the first $r+1$ standard basis vectors for $\mathbb{R}^{2 r+1}$.

The map $L^{\prime}: \mathbb{R}^{2} \rightarrow J_{R}\left(X^{\prime}\right)$ described above is covered by

$$
\ell: \mathbb{R}^{2} \rightarrow V^{t} ; \ell(z, \bar{z})=z r e s_{0} \zeta^{-1}-\bar{z} r e s_{\infty} \zeta
$$

where e.g. $\operatorname{res}_{0} \zeta^{-1}: V \rightarrow \mathbb{C}$ takes the residue of $\zeta^{-1} \omega$ at $\zeta=0$. In terms of the dual basis for $V^{t}$ this has coordinates

$$
\ell: \mathbb{R}^{2} \rightarrow \mathbb{R}^{2 r+1} ; \ell(z, \bar{z})=z U+\bar{z} \bar{U}
$$


where $U \in \mathbb{C}^{2 r+1}$ has coordinates

$$
U=\frac{-1}{2 \pi i}\left(2, \ldots, \alpha_{j}^{-1}+\alpha_{j}, \ldots, i\left(\alpha_{j}^{-1}-\alpha_{j}\right), \ldots\right)
$$

where $\alpha_{j}^{2}=a_{j}$. The map is periodic precisely when there exists $z \in \mathbb{C}$ for which

$$
z U+\bar{z} \bar{U}=\left(p_{0}, p_{1}, \ldots, p_{r}, 0 \ldots, 0\right), p_{j} \in \mathbb{Z} .
$$

If we write $z=x+i y$ then these $2 r+1$ equations become

$$
2 y=-\pi p_{0}, y\left(\alpha_{j}+\alpha_{j}^{-1}\right)=-\pi p_{j}, x\left(\alpha_{j}-\alpha_{j}^{-1}\right)=0, j=1, \ldots, r .
$$

These equations have a solution for $a_{j}<1$ if and only if $x=0$ and

$$
\alpha_{j}^{2}-\frac{2 p_{j}}{p_{0}} \alpha_{j}+1=0 \text {. }
$$

Remark. The reader may be wondering why we only consider $a_{j} \in \mathbb{R}$. The more general case of complex nodes also leads to periodic maps $\varphi: \mathbb{R}^{2} \rightarrow S^{2}$. The conditions are that, writing $a_{j}=r_{j}^{2} e^{2 \theta_{j}}$, there must be positive integers $p_{0}, \ldots, p_{r}$ for which

$$
r_{j}^{2}-\frac{2 p_{j}}{p_{0}} \cos \left(\theta_{j}\right) r_{j}+1=0 .
$$

However, these are not the Gauss maps of periodic CMC surfaces unless $\theta_{j}=$ 0 . Indeed, it is not obvious even then that we obtain periodic CMC surfaces since none of the discussion above accounts for the extra condition that the CMC surface must also have a period when its Gauss map does. That this happens when (14) is satisfied follows from an argument I learned from Martin Kilian and Nick Schmitt, which exploits the dressing construction. Unfortunately to describe this closing argument would take us too far afield, although I will say something about the dressing construction in the next section.

1.8. Two reconstructions of the harmonic map: Symes' method and dressing the vacuum. I know of three approaches to reconstructing the harmonic map from its spectral data. The first of these, which I will describe in a more general context later, boils down to writing the map down in terms of the $\theta$-functions for $X^{\prime}$ (cf. [1]). The other two methods use a loop group and require one to be able to perform a certain loop group factorization. Until recently this had only theoretical interest, but with the advent of Nick Schmitt's CMCLab software it is now possible to perform explicit calculations involving the (approximate) factorization, so I want to take this opportunity to explain how to reproduce the map $\varphi: \mathbb{R}^{2} \rightarrow S^{2}$ (and hence its associated family of CMC surfaces) from its spectral data. Before I begin we must recall some fundamentals about the application of loop groups to the construction of harmonic maps.

First, set $G^{\mathbb{C}}=S L_{2}(\mathbb{C})$ and let $G$ denote its compact real form $S U_{2}$. For $\epsilon \in \mathbb{R}^{+}$with $0<\epsilon<1$ we let $C$ be the union of circles $\{\zeta:|\zeta|=$ 
$\epsilon$ or $\left.|\zeta|=\epsilon^{-1}\right\}$ on the Riemann sphere $\mathbb{C} \cup\{\infty\}$ and consider it as the common boundary of the two open sets

$$
E=\left\{\zeta: \epsilon<|\zeta|<\epsilon^{-1}\right\}, I=\left\{\zeta:|\zeta|<\epsilon \text { or }|\zeta|>\epsilon^{-1}\right\}
$$

We will work with the loop group (of "twisted loops")

$$
\Lambda_{C} G=\left\{C^{\omega} \operatorname{maps} g: C \rightarrow G^{C} \mid g_{\bar{\zeta}^{-1}}=g_{\zeta}^{\dagger}, \nu\left(g_{\zeta}\right)=g_{-\zeta}\right\} .
$$

This loop group contains in particular $\Lambda_{E} G$, the subgroup of those $g \in \Lambda_{C} G$ which extend holomorphically into $E$, and $\Lambda_{I} G$, the subgroup of those $g \in$ $\Lambda_{C} G$ which extend holomorphically into $I$ such that $g_{0}$ is upper triangular with positive real diagonal entries. It is well known (see [16]) that every $g \in \Lambda_{C} G$ factorizes uniquely into $g_{E} g_{I}$ where $g_{E} \in \Lambda_{E} G$ and $g_{I} \in \Lambda_{I} G$ : this is sometimes called the Iwasawa decomposition for $\Lambda_{C} G$.

The relevance of these groups to our harmonic maps can be encapsulated in the following theorem. First, notice that the simplest non-conformal map $\varphi: \mathbb{R}^{2} \rightarrow S^{2}$, which has been dubbed the "vacuum solution", maps onto a great circle and is framed by the homomorphism

$$
F^{(0)}: \mathbb{R}^{2} \rightarrow S U_{2} ; F^{(0)}=\exp (z A-\bar{z} A) ; A=\left(\begin{array}{cc}
0 & 1 \\
1 & 0
\end{array}\right) .
$$

This has extended frame

$$
F_{\zeta}^{(0)}: \mathbb{R}^{2} \rightarrow \Lambda_{E} G ; F_{\zeta}^{(0)}=\exp \left(\zeta^{-1} z A-\zeta \bar{z} A\right) .
$$

Theorem 2. 6, 7] Let $\varphi: \mathbb{R}^{2} \rightarrow S^{2}$ be a non-conformal harmonic map of finite type with polynomial Killing field $\xi_{\zeta}(z)$, in the form (4).

(1) $\varphi$ has an extended frame given by

$$
F_{\zeta}=\exp \left(z \zeta^{2 p} \xi_{\zeta}(0)\right)_{E}
$$

This is "Symes' formula" 6.

(2) For some $0<\epsilon<1$ there exists $g_{\zeta} \in \Lambda_{I} G$ so that $\varphi$ has an extended frame given by

$$
F_{\zeta}=\left(g_{\zeta} F_{\zeta}^{(0)}\right)_{E}
$$

This is "dressing the vacuum solution" [7].

Since in both formulae the frame satisfies the same Maurer-Cartan equations with $F_{\zeta}(0)=I$, each method gives the same extended frame. Now I will describe how to compute the polynomial Killing field $\xi_{\zeta}$ and the dressing matrix $g_{\zeta}$ corresponding to the spectral data $X, \lambda, \mathcal{L}$ for a particularly amenable choice of $\mathcal{L}$.

Proposition 4. Let $X, \lambda$ correspond to the curve with affine equation

$$
y^{2}=\lambda \prod_{j=1}^{g}\left(\lambda-a_{j}\right)\left(1-\bar{a}_{j} \lambda\right) ; 0<\left|a_{j}\right|<1 .
$$


and let $\mathcal{L}=\mathcal{O}_{X}\left(R_{+}\right)$where $R_{+}$is the divisor $P_{0}+\sum_{j=1}^{g} R_{j}$ for $\lambda\left(R_{j}\right)=a_{j}$. Then the non-conformal map $\varphi: \mathbb{R}^{2} \rightarrow S^{2}$ with spectral data $X, \lambda, \mathcal{L}$ arises from:

(1) Symes' formula using $\xi_{\zeta}(0)=\eta_{\zeta}-\eta_{\bar{\zeta}^{-1}}^{\dagger}$ where

$$
\eta_{\zeta}=\left(\begin{array}{cc}
0 & \zeta \prod_{j=1}^{g}\left(1-\bar{a}_{j} \zeta^{2}\right) \\
\zeta \prod_{j=1}^{g}\left(\zeta^{2}-a_{j}\right) & 0
\end{array}\right)
$$

(2) dressing the vacuum solution by

$$
g_{\zeta}=\left(\begin{array}{cc}
h^{-1 / 4} & 0 \\
0 & h^{1 / 4}
\end{array}\right) ; \quad h=\prod_{j=1}^{g}\left(\frac{\zeta^{2}-a_{j}}{1-\bar{a}_{j} \zeta^{2}}\right) .
$$

Proof. 1. Given an orthonormal basis $e_{1}, e_{2}$ for $\Gamma(\mathcal{L})$ we obtain a $B$-module morphism

$$
K:\left\{f \in \mathbb{C}\left[X_{A}\right]: \overline{\rho^{*} f}=-f\right\} \rightarrow\left\{\xi_{\zeta}(z): d \xi=[\xi, \alpha], \quad \xi_{\bar{\zeta}^{-1}}=-\xi_{\zeta}^{\dagger}\right\}
$$

in which each $\xi$ is algebraic (indeed, a Laurent polynomial) in $\lambda$. In fact this map is an isomorphism for real algebraic $\xi$ [19]. It arises from the commutative diagram

$$
\begin{array}{ccc}
\hat{\Gamma}\left(\mathcal{E}_{z, A}(R)\right) & \stackrel{\times f}{\rightarrow} & \hat{\Gamma}\left(\mathcal{E}_{z, A}(R)\right) \\
\epsilon_{z} \downarrow & & \downarrow \epsilon_{z} \\
\hat{B} \otimes \mathbb{C}^{2} & \stackrel{\xi(f)}{\rightarrow} & \hat{B} \otimes \mathbb{C}^{2}
\end{array}
$$

This gives $\xi_{\lambda}(z)=K(f)$ for each $z$, where we recall from (5) that $\xi_{\lambda}=$ $\mathrm{Ad} \kappa \cdot \xi_{\zeta}$. Since $\theta f \theta^{-1}=f$ it follows, by combining this diagram and the diagram (11), that $\xi(0)=F \xi(z) F^{-1}$ whence $d \xi=[\xi, \alpha]$. For the purposes of Symes' formula we want to compute $K(f)$ at $z=0$ for $f=y-\overline{\rho_{*} y}$. Since

$$
K\left(\overline{\rho_{*} y}\right)={\overline{\rho_{*} K(y)}}^{t}
$$

it suffices to compute $K(y)$ at $z=0$. A simple computation shows that with respect to the trace inner product (9) $\Gamma\left(\mathcal{O}_{X}\left(R_{+}\right)\right)$has an orthonormal basis given by

$$
e_{1}=\frac{y}{\lambda \prod_{j=1}^{g}\left(\lambda-a_{j}\right)}, \quad e_{2}=1 .
$$

Here we are identifying holomorphic sections of $\mathcal{L}$ with rational functions on $X$ whose divisor of poles is no worse than $R_{+}$. Notice that $e_{1}$ generates $\Gamma\left(\mathcal{L}\left(-P_{\infty}\right)\right)$ while $e_{2}$ generates $\Gamma\left(\mathcal{L}\left(-P_{0}\right)\right)$. Now $K(y)$ is the matrix

$$
\left(\begin{array}{ll}
\alpha & \beta \\
\gamma & \delta
\end{array}\right)
$$

where

$$
y e_{1}=\alpha e_{1}+\beta e_{2}, \quad y e_{2}=\gamma e_{1}+\delta e_{2}
$$


so that at $z=0$

$$
K(y)=\left(\begin{array}{cc}
0 & \prod_{j=1}^{g}\left(1-\bar{a}_{j} \lambda\right) \\
\lambda \prod_{j=1}^{g}\left(\lambda-a_{j}\right) & 0
\end{array}\right) .
$$

Finally, let $\eta_{\zeta}$ be the twisted loop $\mathrm{Ad} \kappa^{-1} \cdot K(y)$ to obtain the formula (15). 2. Let us consider the geometric meaning of the equation (16). If we write these loops in their untwisted form then $g_{\lambda} F_{\lambda}^{(0)}=F_{\lambda} b_{\lambda}$ where $F_{\lambda}$ extends holomorphically to an annulus on the $\lambda$-sphere (which we will call $E$ despite the abuse of notation) and $b_{\lambda}$ extends holomorphically to a pair of discs about $\lambda=0, \infty$ (which we will call $I$ ) and is upper triangular at $\lambda=0$. A little thought shows that the columns of $F^{-1 t}$ represent $e_{1}^{z} \theta^{-1}, e_{2}^{z} \theta^{-1}$, thought of as sections of the rank two vector bundle $\lambda_{*} \mathcal{L}$ over $E$, with respect to the global frame $e_{1}^{0}, e_{2}^{0}$. Let $\phi_{E}$ denote the trivialisation of $\lambda_{*} \mathcal{L}$ determined by this global frame, then the equation $g_{\lambda}^{-1 t} b_{\lambda, z=0}^{-1 t}=I$ expresses the fact that there is some local trivialisation $\phi_{I}$ for $\lambda_{*} \mathcal{L}$ over $I$ for which the transition relation on $E \cap I$ is

$$
g^{-1 t} \phi_{I}=\phi_{E} .
$$

Therefore $g_{\lambda}^{t}$ is the matrix whose columns are $\phi_{I}\left(e_{1}^{0}\right), \phi_{I}\left(e_{2}^{0}\right)$. Now we recall from 15 that $\phi_{I}$ is obtained by direct image from a trivialisation of $\mathcal{L}$ over $\lambda^{-1}(I)$ in the following way. Let $s_{I}$ be a non-vanishing holomorphic section of $\mathcal{L}$ over $\lambda^{-1}(I)$. By definition $\Gamma\left(I, \lambda_{*} \mathcal{L}\right)=\Gamma\left(\lambda^{-1}, \mathcal{L}\right)$ and $s_{I}$ induces the trivialisation

$$
\phi_{I}: \Gamma\left(I, \lambda_{*} \mathcal{L}\right) \rightarrow \operatorname{Hol}\left(I, \mathbb{C}^{2}\right) ; \quad s \mapsto\left(s_{1}, s_{2}\right)
$$

where $s / s_{I}=s_{1}\left(\zeta^{2}\right)+\zeta s_{2}\left(\zeta^{2}\right)$. Any trivialisation $\phi_{I}$ obtained this way and which gives $\operatorname{det}\left(g_{\lambda}\right)=1$ will provide a suitable matrix $g_{\lambda}$ (the freedom here is right multiplication of $g_{\lambda}$ by any element of $\Lambda_{I} G$ which commutes with $F_{\lambda}^{(0)}$ for all $z$ ). To calculate $g_{\lambda}$ we let $e_{1}, e_{2}$ be the basis (17) and initially take $s_{I}=e_{1}$ : this is appropriate since as a function it has a simple pole at $P_{0}$ and does not vanish at $P_{\infty}$, therefore it represents a non-vanishing section of $\mathcal{L}$ over $I$ provided $I$ is small enough. Now we write

$$
e_{1} / e_{1}=1+\zeta .0, \quad e_{2} / e_{1}=0+\zeta . \prod_{j=1}^{g}\left(\frac{\zeta^{2}-a_{j}}{1-\bar{a}_{j} \zeta^{2}}\right)^{1 / 2} .
$$

However, this choice of $s_{I}$ does not give $\operatorname{det}\left(g_{\lambda}\right)=1$, so it remains to rescale $s_{I}$ by the appropriate non-vanishing function to obtain (16).

Remark 1. For simplicity define $\bar{\eta}_{\zeta}=-\eta_{\bar{\zeta}^{-1}}^{\dagger}$. It suffices to use $\bar{\eta}_{\zeta}$ instead of $\xi_{\zeta}(0)$ in Symes' formula, since $[\eta, \bar{\eta}]=0$ and $\exp \left(z \zeta^{2 g} \eta_{\zeta}\right)_{E}=I$ (since $\eta_{\zeta}$ is polynomial in $\zeta)$, therefore $\exp \left(z \zeta^{2 g} \xi_{\zeta}(0)\right)_{E}=\exp \left(z \zeta^{2 g} \bar{\eta}_{\zeta}\right)_{E}$. Moreover, by combining the extended frame with the Sym-Bobenko formula [2, 14] we can produce CMC tori once we know a choice of branch points for the spectral curve which satisfies the double periodicity condition (not just the periodicity condition above, which only makes the Gauss map periodic, but 

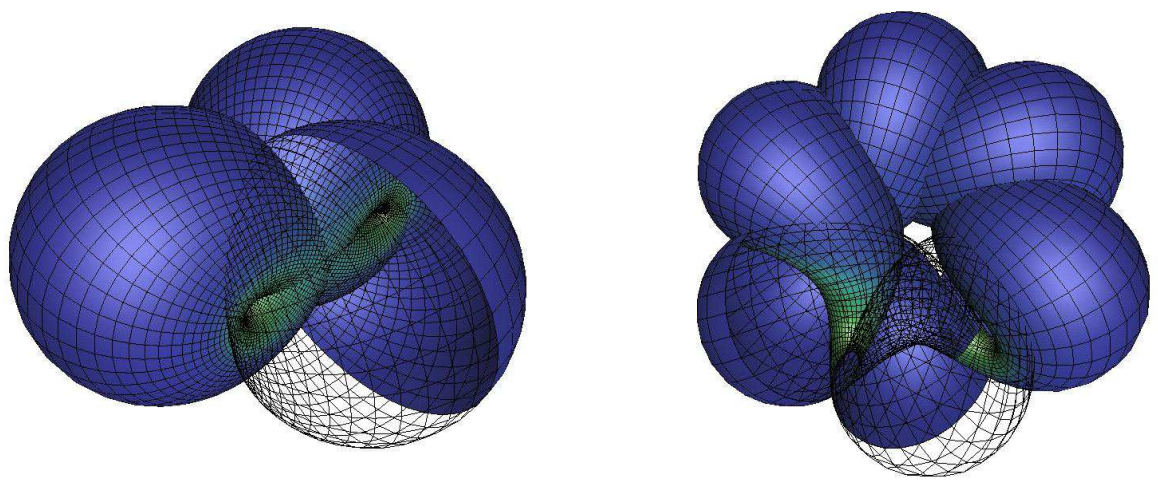

Figure 1. Wente torus (left), twisty torus (right).

the full CMC periodicity condition described in [1]). The following examples for a genus two curve are due to Matthias Heil (private communication):

$$
\begin{aligned}
& a_{1}=0.1413+0.1018 i, a_{2}=0.1413-0.1018 i, \text { (Wente torus); } \\
& a_{1}=0.124+0.1485 i, a_{2}=0.4387-0.071 i \text { (twisty torus). }
\end{aligned}
$$

The corresponding CMC tori are drawn in figure 1.

Remark 2. In fact we can use the dressing construction to produce all harmonic maps with spectral data $X, \lambda$. For even though the dressing matrix (16) corresponds to the line bundle $\mathcal{O}_{X}\left(R_{+}\right)$every other line bundle satisfying the reality condition is of the form $\mathcal{O}_{X}\left(R_{+}\right) \otimes L$ where $L \in J_{R}(X)$. It was shown in [17] that the full family of these is swept out by the "higher flows" described in [7]. That means an extended frame for the harmonic map with data $X, \lambda, \mathcal{O}_{X}\left(R_{+}\right) \otimes L$ is given by dressing the vacuum by

$$
g_{\zeta} \exp \left(\sum_{j=1}^{\infty}\left(t_{j} \zeta^{j} A^{j}-\bar{t}_{j} \zeta^{-j} A^{-j}\right)\right)
$$

for some sequence $t_{j} \in \mathbb{C}$. Moreover, for a map of finite type only finitely many of the higher flows are independent, so there is no need for an infinite sum here. It can be shown that it suffices to have only $t_{1}, t_{3}, \ldots, t_{2 g-1}$ taking any values and all other parameters zero: the first flow $t_{1}$ is just a $z$-translation of the surface domain.

Remark 3. By combining proposition 3 with proposition 4 we can compute the one and two bubbletons in figure 2 . These have respectively $r=1, g=2$ and $r=2, g=4$. Using the previous remark we obtain a real $g$-parameter family of deformations of these surfaces. Each bubble can be moved relative to any other (or the cylinder) by a translation along the cylinder or a rotation about its circumference. Thus each bubble contributes two real parameters: there are $r$ bubbles altogether. This demonstrates that $J_{R}\left(X^{\prime}\right) \cong\left(\mathbb{R} \times S^{1}\right)^{r}$. It is interesting to note that we can also think of the bubbletons as being 


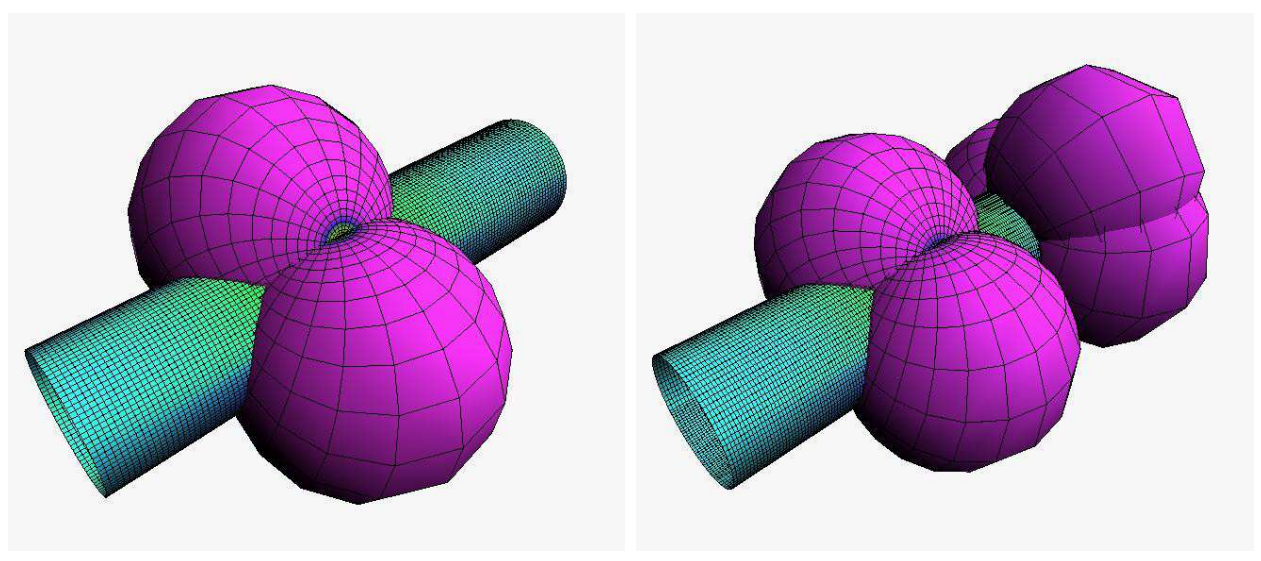

Figure 2. One bubbleton and two bubbleton.

constructed by dressing the vacuum by a rational loop on the $\zeta$-sphere. We can re-scale the matrix $g_{\zeta}$ in (16) so that for a nodal curve (13) it becomes

$$
\prod_{j=1}^{r}\left(\begin{array}{cc}
\bar{a}_{j} \zeta^{2}-1 & 0 \\
0 & \zeta^{2}-a_{j}
\end{array}\right), r=g / 2 .
$$

This dressing matrix produces the same surface and resembles a product of Bäcklund transforms in the sense of 28 . Although proposition 3 only proves that the Gauss map of the CMC surface is periodic (given the conditions (14) on each $a_{j}$ ) it turns out that the CMC surface itself is periodic. This can be shown by examining the effect on the monodromy matrix of $F_{\zeta}^{(0)}$ of dressing by any factor in the product (18). This approach was explained to me by Martin Kilian and Nick Schmitt. Their approach also explains the geometric significance of the positive integers $p_{0}, \ldots, p_{n}$ appearing in (14). The integer $p_{0}$ determines the number of times the cylindrical end of the bubbleton wraps around itself, while $p_{j}$ is the number of "lobes" the $j$-th bubble possesses.

\section{HARMONiC AND PluRI-HARMONIC MAPS INTO $G r_{k}\left(\mathbb{C}^{n+1}\right)$.}

Let $k \leq(n+1) / 2$. Here I will briefly recount the theory given in [20] for constructing pluri-harmonic maps $\varphi: \mathbb{R}^{2 k} \rightarrow G r_{k}\left(\mathbb{C}^{n+1}\right)$ which generalizes the construction given above (recall that a map is pluri-harmonic if it is harmonic on any holomorphic curve: here $\mathbb{R}^{2 k}$ is given the usual complex structure). At the end I will illustrate this with the example $X \cong \mathbb{P}^{1}$.

To begin, the spectral data here consists of a (smooth, we will assume) compact Riemann surface $X$ (of genus $g$ ) with real involution $\rho$ together with a degree $n+1$ function $\lambda$ on $X$ and a line bundle $\mathcal{L}$ over $X$. We require: $\overline{\rho_{*} \lambda}=\lambda^{-1}$; the ramification divisor $R$ of $\lambda$ has no support over $|\lambda|=1$; and $\rho$ fixes every point over $|\lambda|=1$. In that case $R=R_{+}+\rho_{*} R_{+}$, where $R_{+}$ is the divisor of ramification over $|\lambda|>1$. We can choose $\mathcal{L}$ to satisfy the 
reality condition $\overline{\rho_{*} \mathcal{L}} \cong \mathcal{L}^{t}(R)$ by taking any element of the compact real connected $g$-dimensional torus

$$
\mathcal{N}=\left\{\mathcal{O}_{X}\left(R_{+}\right) \otimes L: L \in J_{R}\right\}
$$

where $J_{R}$ is the identity component of $\left\{L \in J(X): L \cong{\overline{\rho_{*} L}}^{-1}\right\}$. It can be shown that for any such bundle $\lambda_{*} \mathcal{L}$ is a trivial rank $n+1$ bundle so $\operatorname{dim}(\Gamma(\mathcal{L}))=n+1$. Further, the trace pairing equips $\Gamma(\mathcal{L})$ with a Hermitian inner product.

As before, the geometry of the construction is best understood by working with the singularisation $X^{\prime}$ of $X$ obtained by identifying the $n+1$ points $O_{1}, \ldots, O_{n+1}$ lying over $\lambda=1$ together to obtain a nodal singularity $O$ on $X^{\prime}$. A line bundle $\mathcal{L}^{\prime}$ over $X^{\prime}$ is best thought of as a line bundle $\mathcal{L}$ over $X$ equipped with a linear identification of the fibres over $O_{1}, \ldots, O_{n+1}$ : we can think of this as assigning a non-zero element to each stalk $\mathcal{L} \mid O_{j}$. In $\operatorname{Pic}\left(X^{\prime}\right)$ (the algebraic group of all holomorphic line bundles over $X^{\prime}$ ) we distinguish the real variety

$$
\mathcal{N}^{\prime}=\left\{\mathcal{O}_{X^{\prime}}\left(R_{+}\right) \otimes L^{\prime}: L^{\prime} \in J_{R}^{\prime}\right\},
$$

where $J_{R}^{\prime}=\left\{L^{\prime} \in J\left(X^{\prime}\right): L^{\prime} \cong{\overline{\rho_{*} L^{\prime}}}^{-1}\right\}$. Let $\pi: \operatorname{Pic}\left(X^{\prime}\right) \rightarrow \operatorname{Pic}(X)$ be the natural epimorphism for which $\pi\left(\mathcal{L}^{\prime}\right)=\mathcal{L}$. An element of $\Gamma\left(\mathcal{L}^{\prime}\right)$ is a global section of $\mathcal{L}$ which "takes the same value" at each $O_{j}$ using the fibre identification with which $\mathcal{L}^{\prime}$ is equipped. For $\mathcal{L}^{\prime} \in \mathcal{N}^{\prime}$, since $\lambda_{*} \mathcal{L}$ is trivial, there is no non-zero global section of $\mathcal{L}$ which vanishes at every $O_{j}$, therefore $\operatorname{dim}\left(\Gamma\left(\mathcal{L}^{\prime}\right)\right)=1$. Thus any non-zero global section of $\mathcal{L}^{\prime}$ gives us a convenient representation for the fibre identification carried by $\mathcal{L}^{\prime}$.

Over $\mathcal{N}^{\prime}$ there exists a natural rank $n+1$ bundle $E^{\prime}$ whose fibre at $\mathcal{L}^{\prime}$ is $\Gamma(\mathcal{L})$. For any $k$ the Grassmann bundle $G r_{k}\left(E^{\prime}\right)$ possesses a canonical trivialisation given pointwise as follows. Let

$$
\left[e_{1} \wedge \ldots \wedge e_{k}\right] \in G r_{k}\left(E^{\prime}\right)
$$

By taking any non-zero $s_{\mathcal{L}} \in \Gamma\left(\mathcal{L}^{\prime}\right)$ we can identify

$$
e_{j} \mapsto v_{j}=\left(\frac{e_{j} \mid O_{1}}{s_{\mathcal{L}} \mid O_{1}}, \ldots, \frac{e_{j} \mid O_{n+1}}{s_{\mathcal{L}} \mid O_{n+1}}\right) \in \mathbb{C}^{n+1}
$$

and this is projectively dependent only on $\mathcal{L}^{\prime}$. Thus we have a natural map

$$
\left[e_{1} \wedge \ldots \wedge e_{k}\right] \mapsto\left[v_{1} \wedge \ldots \wedge v_{k}\right] \in G r_{k}\left(\mathbb{C}^{n+1}\right) .
$$

The relevance of this is that by taking a suitable section of $G r_{k}\left(E^{\prime}\right)$ and applying this trivialisation we obtain a map $J_{R}^{\prime} \cong \mathcal{N}^{\prime} \rightarrow G r_{k}\left(\mathbb{C}^{n+1}\right)$ whose restriction to suitable subgroups of $J_{R}^{\prime}$ is (pluri)-harmonic. This result is true for any choice of isomorphism $J_{R}^{\prime} \cong \mathcal{N}^{\prime}$, so in fact we obtain not just one map but a family of them - these correspond to the deformations made available by the higher flows discussed earlier.

Now I must explain which section of $G r_{k}\left(E^{\prime}\right)$ yields (pluri)-harmonic maps. Although we could discuss the construction of maps of any isotropy 
order we will stick with the simplest case of lowest isotropy order i.e. nonconformal maps. For this we take $\lambda$ to have (at least) $k$ double zeroes $P_{1}, \ldots, P_{k}$. Consequently the divisor of $\lambda$ has the form

$$
(\lambda)=2 P_{1}+\ldots+2 P_{k}+E_{0}-2 Q_{1}-\ldots 2 Q_{k}-E_{\infty}
$$

where $E_{0}, E_{\infty}$ are positive divisors of degree $n+1-2 k$. Let $D_{\infty}$ denote the positive divisor $Q_{1}+\ldots Q_{k}+E_{\infty}$ of degree $n+1-k$, then $D_{\infty}$ gives us a section of $G_{k}\left(E^{\prime}\right)$ by assigning to each $\mathcal{L}^{\prime}$ the $k$-plane $\Gamma\left(\mathcal{L}\left(-D_{\infty}\right)\right)$. Thus by our canonical trivialisation we have map

$$
\psi: J_{R}^{\prime} \cong \mathcal{N}^{\prime} \rightarrow G r_{k}\left(\mathbb{C}^{n+1}\right) .
$$

Now let $\gamma: \mathbb{R}^{2 k} \rightarrow J_{R}^{\prime}$ be the real homomorphism uniquely determined up to scalings by:

$$
\partial \gamma / \partial z_{j}=\partial \mathcal{A}_{P_{j}}^{\prime} / \partial \zeta_{P_{j}}
$$

where $z_{1}, \ldots, z_{k}$ denote complex coordinates on $\mathbb{R}^{2 k}, \zeta_{P_{j}}$ is a local coordinate about $P_{j}$ and $\mathcal{A}_{P_{j}}^{\prime}$ denotes the Abel map for $X^{\prime}$ with base point $P_{j}$.

Theorem 3. 20] The map $\varphi=\psi \circ \gamma: \mathbb{R}^{2 k} \rightarrow G r_{k}\left(\mathbb{C}^{n+1}\right)$ given above is pluri-harmonic. Indeed, the harmonic map obtained by restriction of $\varphi$ to the complex line with tangent $\sum a_{j} \partial / \partial z_{j}$ is harmonic: it is also nowhere conformal iff $\sum a_{j}^{2} \neq 0$.

Remark. According to 17, 18, this theorem accounts for all non-conformal harmonic maps $\varphi: \mathbb{R}^{2} \rightarrow \mathbb{C P}^{n}$ of semisimple finite type (and therefore all non-conformal tori). Indeed I believe it will account for all maps of semisimple finite type into $G r_{k}\left(\mathbb{C}^{n+1}\right)$ using a similar argument. The main unanswered question is to what extent the non-conformal (or more generally, non-isotropic) harmonic tori in $G r_{k}\left(\mathbb{C}^{n+1}\right)$ are accounted for by the tori of semisimple finite type. Some progress has been made in this direction (see [29]) but the problem is not yet settled.

2.1. Explicit formulae in terms of Riemann $\theta$-functions. In the construction above there is, up to scalings, a natural basis $e_{1}, \ldots, e_{k}$ for the $k$-plane $\Gamma\left(\mathcal{L}\left(-D_{\infty}\right)\right.$. For each $j=1, \ldots, k$ let $D_{j}$ be the positive divisor $D_{\infty}+\sum_{k \neq j} Q_{j}$, which has degree $n$, and notice that for any $j$ the divisor of poles of $\lambda$ is $D_{j}+Q_{j}$. Since $\lambda_{*} \mathcal{L}$ is trivial the subspace $\Gamma\left(\mathcal{L}\left(-D_{j}\right) \subset\right.$ $\Gamma\left(\mathcal{L}\left(-D_{\infty}\right)\right)$ is one dimensional and $\mathcal{L}\left(-D_{j}\right)$ is non-speciali of degree $g$. This means we can obtain a non-zero section of it using Riemann's $\theta$-function. To obtain a formula for $\psi$ we then have to understand the behaviour of the fibre identifications. It turns out that these can be incorporated by pulling the $\theta$-line bundle over $J(X)$ back to $J\left(X^{\prime}\right)$ using $\pi$. An explicit formula for $\psi$ is then obtained as follows. Throughout this discussion we take $\mathcal{L}=\mathcal{O}_{X}\left(R_{+}\right)$: any other choice of $\mathcal{L}$ simply amounts to a translation in the argument of the $\theta$-function with no loss of generality.

We know that we can make identifications

$$
J\left(X^{\prime}\right) \cong H^{0}\left(\Omega_{X}(\mathfrak{o})\right)^{t} / H_{1}(X \backslash \mathfrak{o}, \mathbb{Z}) \simeq \mathbb{C}^{g+n} / \Lambda^{\prime},
$$


where $\Omega_{X}(\mathfrak{o})$ is the sheaf of mermorphic differentials on $X$ with divisor of poles no worse than $\mathfrak{o}=O_{1}+\ldots+O_{n+1}$ and $\Lambda^{\prime}$ is a lattice on $2 g+n$ generators. We choose coordinates so that $\pi: J\left(X^{\prime}\right) \rightarrow J(X)$ is covered by the map

$$
\pi: \mathbb{C}^{g+n} \rightarrow \mathbb{C}^{g} ; \quad \tilde{W}=\left(w_{1}, \ldots, w_{g+n}\right) \mapsto W=\left(w_{1}, \ldots, w_{g}\right) .
$$

Now let us define $\theta_{0}(\tilde{W})=\theta(W)$ and for $j=1, \ldots, n$ define

$$
\theta_{j}(\tilde{W})=\exp \left(2 \pi i w_{g+j}\right) \theta\left(W+\mathcal{A}\left(O_{j+1}-O_{1}\right)\right)
$$

where $\theta$ is the classical Riemann $\theta$-function on $\mathbb{C}^{g}$ corresponding to the induced isomorphism $J(X) \simeq \mathbb{C}^{g} / \pi\left(\Lambda^{\prime}\right)$. Each of $\theta_{0}, \ldots, \theta_{n}$ represents a global holomorphic section of the pullback by $\pi$ of the $\theta$-line bundle over $J(X)$ [20].

For $l=1, \ldots, k$ let $\tilde{D}_{l}$ be the unique positive divisor (of degree $g$ ) in the linear system of $\mathcal{L}\left(-D_{l}\right)$ and let $\kappa_{l} \in \mathbb{C}^{g}$ be the appropriate translation for which $\theta\left(\mathcal{A}(P)+\kappa_{l}\right)$ has divisor of zeroes $\tilde{D}_{l}$. Finally, let $f_{l}$ be a rational function on $X$ with divisor

$$
\left(f_{l}\right)=R_{+}-D_{l}-\tilde{D}_{l}
$$

so that $f_{l}(P) \theta\left(\mathcal{A}(P)+\kappa_{l}\right)$ has divisor $R_{+}-D_{l}$.

Proposition 5. 20] Let $v_{l}: \mathbb{C}^{g+n} \rightarrow \mathbb{C}^{n+1}$ be defined by

$$
v_{l}(\tilde{W})=\left(f_{l}\left(O_{1}\right) \theta_{0}\left(\tilde{W}+\kappa_{l}\right), \ldots, f_{l}\left(O_{n+1}\right) \theta_{n}\left(\tilde{W}+\kappa_{l}\right)\right)
$$

Then, taking the base point $\mathcal{O}_{X^{\prime}}\left(R_{+}\right)$on $\mathcal{N}^{\prime}$ for the identification $J_{R}^{\prime} \cong \mathcal{N}^{\prime}$, the map $\psi: J_{R}^{\prime} \rightarrow G r_{k}\left(\mathbb{C}^{n+1}\right)$ above is explicitly given by the $\Lambda^{\prime}$-periodic map

$$
\psi(\tilde{W})=\left[v_{1}(\tilde{W}) \wedge \ldots \wedge v_{k}(\tilde{W})\right] .
$$

An explicit formula for the function $f$ can be obtained using Fay's prime form (see e.g. 23 ). It remains to combine this with the real homomorphism $\gamma: \mathbb{R}^{2 k} \rightarrow \mathbb{C}^{g+n} / \Lambda^{\prime}$ which we have essentially computed earlier (cf. [20]). For illustration I will do these calculations explicitly for $X \cong \mathbb{P}^{1}$ in the next section.

2.2. Example: $X$ is the Riemann sphere. Let $\zeta$ be a rational parameter on $X \cong \mathbb{P}^{1}$ and define the real involution to be $\rho_{*} \zeta=\bar{\zeta}^{-1}$, then to satisfy all our conditions $\lambda$ must be of the form

$$
\lambda=\alpha \prod_{j=1}^{k} \frac{\left(\zeta-P_{j}\right)^{2}}{\left(\zeta-\bar{P}_{j}^{-1}\right)^{2}} \prod_{i=1}^{n+1-2 k} \frac{\left(\zeta-E_{j}\right)}{\left(\zeta-\bar{E}_{j}^{-1}\right)},
$$

where the points $P_{1}, \ldots, P_{k}, E_{1}, \ldots, E_{n+1-2 k}$ all lie inside $|\zeta|<1$ (cf. [27]). The constant $\alpha$ is chosen so that $|\lambda|=1$ over $|\zeta|=1$.

First we construct the homomorphism $\gamma: \mathbb{R}^{2 k} \rightarrow J_{R}^{\prime}$. To fix the isomorphism (19) we choose the basis $\omega_{1}, \ldots, \omega_{n}$ of $H^{0}(\Omega(\mathfrak{o}))$ given by

$$
\omega_{m}=\frac{1}{2 \pi i}\left(\frac{1}{\zeta-O_{m+1}}-\frac{1}{\zeta-O_{1}}\right) d \zeta, \quad m=1, \ldots, n .
$$


Let $a_{m} \in H_{1}(X \backslash \mathfrak{o}, \mathbb{Z})$ be the class of a positively oriented cycle about $O_{m+1}$ only, so that $\oint_{a_{l}} \omega_{m}=\delta_{l m}$. With these bases we have

$$
J\left(X^{\prime}\right) \cong \mathbb{C}^{n} / \mathbb{Z}^{n} \stackrel{\exp (2 \pi i \cdot)}{\rightarrow}\left(\mathbb{C}^{*}\right)^{n} .
$$

Take $\zeta_{P_{j}}=\zeta-P_{j}$ for the local parameter about $P_{j}$ and recall from earlier that as an element of $H^{0}(\Omega(\mathfrak{o}))^{t} \cong T_{0} J\left(X^{\prime}\right)$

$$
\frac{\partial \mathcal{A}_{P_{j}}^{\prime}}{\partial \zeta_{P_{j}}}: \omega_{m} \mapsto \operatorname{res}_{P_{j}} \zeta_{P_{j}}^{-1} \omega_{m} .
$$

In our coordinates this is the vector $\frac{1}{2 \pi i} U_{j}$ where $U_{j} \in \mathbb{C}^{n}$ has $m$-th coordinate

$$
U_{j m}=\frac{1}{P_{j}-O_{m}}-\frac{1}{P_{j}-O_{1}} .
$$

The map $\gamma: \mathbb{R}^{2 k} \rightarrow\left(\mathbb{C}^{*}\right)^{n}$ is given by

$$
\gamma\left(z_{1}, \ldots, z_{k}\right)=\exp \left(\sum_{j=1}^{k}\left(U_{j} z_{j}-\bar{U}_{j} \bar{z}_{j}\right)\right) .
$$

Now to apply proposition 5 we notice that since $J(X)$ is the trivial group we can take $\theta \equiv 1$. So for $\tilde{W}=\left(w_{1}, \ldots, w_{n}\right)$ we have simply

$$
\theta_{0}(\tilde{W})=1, \theta_{1}(\tilde{W})=\exp \left(2 \pi i w_{1}\right), \ldots, \theta_{n}(\tilde{W})=\exp \left(2 \pi i w_{n}\right) .
$$

Finally, we need the divisors

$$
D_{l}=2 Q_{1}+\ldots+Q_{l}+\ldots 2 Q_{k}+E_{\infty}, \quad l=1, \ldots, k,
$$

where $Q_{j}=\bar{P}_{j}^{-1}$ and $E_{\infty}=\bar{E}_{1}^{-1}+\ldots+\bar{E}_{n+1-2 k}^{-1}$. Let $f_{l}$ be any rational function with divisor $R_{+}-D_{l}$ and define $v_{l}: \mathbb{C}^{k} \rightarrow \mathbb{C}^{n+1}$ by

$$
v_{l}\left(z_{1}, \ldots, z_{k}\right)=\left(f_{l}\left(O_{1}\right), f_{l}\left(O_{2}\right) \gamma_{1}, \ldots, f_{l}\left(O_{n+1}\right) \gamma_{n}\right)
$$

where $\gamma_{m}=\exp \left(\sum_{j=1}^{k}\left(z_{j} U_{j m}-\bar{z}_{j} \bar{U}_{j m}\right)\right.$.

Proposition 6. 20] The pluri-harmonic map $\varphi: \mathbb{R}^{2 k} \rightarrow G r_{k}\left(\mathbb{C}^{n+1}\right)$ with spectral data $X \cong \mathbb{P}^{1}$ and $\lambda$ given by (20) is given by

$$
\varphi\left(z_{1}, \ldots, z_{k}\right)=\left[v_{1} \wedge \ldots \wedge v_{k}\right] .
$$

This map is totally equivariant i.e. it can be framed by a homomorphism $\mathbb{R}^{2 k} \rightarrow U_{n+1}$.

By a result of Kenmotsu 13 (see also 3]) the minimal (i.e. conformal harmonic) totally equivariant maps $\mathbb{R}^{2} \rightarrow \mathbb{C P}^{n}$ include those minimal totally real maps which are isometric for the flat metric on $\mathbb{R}^{2}$. A study of their periodicity can be found in [12. To pass from non-conformal to conformal maps in our construction (in the case $k=1$ i.e. $\mathbb{C P}^{n}$ ) one insists that $\lambda$ has a zero of degree 3 at $P_{1}$. In particular, this requires $n \geq 2$. 
Remark. There is a geometric interpretation behind the form of $v_{l}$. Suppose $\varphi: \mathbb{R}^{2 k} \rightarrow G r_{k}\left(\mathbb{C}^{n+1}\right)$ is totally equivariant with frame

$$
F=\exp \left(z \cdot A-\bar{z} \cdot A^{\dagger}\right), \quad z \cdot A=\sum_{j=1}^{k} z_{j} A_{j},
$$

where $A_{1}, \ldots, A_{k} \in \mathfrak{g l}_{n+1}(\mathbb{C})$ are mutually commuting normal matrices. We will assume $\varphi$ is based so that $\varphi(0)=\left[e_{1} \wedge \ldots \wedge e_{k}\right]$ where the $e_{j}$ are the standard basis vectors for $\mathbb{C}^{n+1}$. The matrices $A_{j}$ and their Hermitian transposes may be simultaneously diagonalized by a unitary matrix: $M A_{j} M^{-1}=D_{j}$ where $M$ is unitary and each $D_{j}$ is diagonal. Therefore

$$
\begin{aligned}
M \circ \varphi & =M F M^{-1} M \circ\left[e_{1} \wedge \ldots \wedge e_{k}\right] \\
& =\exp (z \cdot D-\bar{z} \cdot \bar{D}) \circ\left[u_{1} \wedge \ldots \wedge u_{k}\right]
\end{aligned}
$$

where $u_{1}, \ldots, u_{k}$ are the first $k$ columns of $M$. Thus

$$
M \circ \varphi=\left[v_{1} \wedge \ldots \wedge v_{k}\right]
$$

where $v_{l}=\exp (z \cdot D-\bar{z} \cdot \bar{D}) \circ u_{l}$. Notice that this is essentially the form of the map we derived above, using

$$
D_{j}=\operatorname{diag}\left(1, U_{j 1}, \ldots, U_{j n}\right), j=1, \ldots, k .
$$

The $v_{l}$ appearing in (21) span the same $k$-plane as these but are not necessarily orthonormal.

\section{REFERENCES}

[1] A I Bobenko, All constant mean curvature tori in $R^{3}, S^{3}$ and $H^{3}$ in terms of thetafunctions, Math. Ann. 290 (1991), 209-245.

[2] A I Bobenko, Constant mean curvature surfaces and integrable equations, Uspekhi Mat. Nauk 46:4 (1991), 3-42; Russian Math. Surv. 46:4 (1991), 1-45;

[3] J Bolton \& L Woodward, Minimal surfaces in $\mathbb{C P}^{n}$ of constant curvature and Kähler angle, Math. Proc. Camb. Phil. Soc. 112 (1992), 287-296.

[4] F E Burstall, Harmonic tori in spheres and complex projective spaces, J. reine. angew. Math. 469 (1995), 149-177.

[5] F E Burstall, D Ferus, F Pedit, \& U Pinkall,Harmonic tori in symmetric spaces and commuting Hamiltonian systems on loop algebras, Ann. of Math. 138 (1993), 173-212.

[6] F E Burstall \& F Pedit, Harmonic maps via Adler-Kostant-Symes theory, in Harmonic maps and integrable systems, ed: A P Fordy \& J C Wood, Aspects of Mathematics E23, Vieweg 1994.

[7] F E Burstall \& F Pedit, Dressing orbits of harmonic maps, Duke Math. J. 80 (1995), 353-382.

[8] N Ercolani, H Knörrer \& E Trubowitz, Hyperelliptic curves that generate constant mean curvature tori in $\mathbb{R}^{3}$, Integrable Systems (Luminy, 1991) 81-114, Prog. in Math. 115, Birkhäuser, Boston 1993.

[9] D Ferus, F Pedit, U Pinkall \& I Sterling, Minimal tori in $S^{4}$, J. reine angew. Math. 429 (1992), 1-47.

[10] N J Hitchin, Harmonic maps from a 2-torus to the 3-sphere, J. Diff. Geom. 31 (1990), 627-710.

[11] C Jaggy, On the classification of constant mean curvature tori in $\mathbb{R}^{3}$, Comment. Math. Helv. 69 (1994), 640-658. 
[12] G Jensen \& R Liao, Families of flat minimal tori in $\mathbb{C P}^{n}$, J. Diff. Geom. 42 (1995), 113-132.

[13] K Kenmotsu, On minimal immersions of $\mathbb{R}^{2}$ into $\mathbb{P}^{n}(\mathbb{C})$, J. Math. Soc. Japan 37 (1985), 665-682.

[14] M Kilian, I McIntosh \& N Schmitt, New constant mean curvature surfaces, Exp. Math. 9:4 (2000), 595-611.

[15] I McIntosh, Global solutions of the elliptic $2 D$ periodic Toda lattice, Nonlinearity 7 (1994), 85-108.

[16] I McIntosh, Infinite dimensional Lie groups and the two-dimensional Toda lattice, in Harmonic maps and integrable systems, ed: A P Fordy \& J C Wood, Aspects of Mathematics E23, Vieweg 1994.

[17] I McIntosh, A construction of all non-isotropic harmonic tori in complex projective space, Int. J. Math. 6 (1995), 831-879.

[18] I McIntosh, Two remarks on the construction of harmonic tori in $\mathbf{C P}^{n}$, Int. J. Math. 7 (1996), 515-520.

[19] I McIntosh, On the existence of superconformal 2-tori and doubly periodic affine Toda fields, J. Geometry Phys. 24 (1998), 223-243.

[20] I McIntosh, Harmonic tori and generalised Jacobi varieties, Comm. Anal. Geom. 9 (2001), 423-449.

[21] M Melko \& I Sterling, Integrable systems, harmonic maps and the classical theory of surfaces, in Harmonic maps and integrable systems, ed: A P Fordy \& J C Wood, Aspects of Mathematics E23, Vieweg 1994.

[22] D Mumford, An algebro-geometric construction of commuting operators and of solutions to the Toda lattice equation, Kortweg de Vries equation and related non-linear equations, Intl. Symp. on Algebraic Geometry, Kyoto 1977, 115-153.

[23] D Mumford, Tata lectures on theta II, Birkhäuser, Boston 1983.

[24] U Pinkall \& I Sterling, On the classification of constant mean curvature tori, Ann. Math. 130 (1989), 407-451.

[25] A Reimann \& M Semenov-Tian-Shansky, Reduction of hamiltonian systems, affine Lie algebras and Lax equations, II, Invent. Math. 63(1981), 423-432.

[26] J-P Serre, Algebraic groups and class fields, Graduate Texts in Math. 117, Springer, New York (1988).

[27] T Taniguchi, Non-isotropic harmonic tori in complex projective spaces and configurations of points on Riemann surfaces, Tohoku Math. Publ. 14 (1999).

[28] C-L Terng \& K Uhlenbeck, Bäcklund transformations and loop group actions. Comm. Pure. Appl. Math. 53 (2000), 1-75.

[29] S Udagawa, Harmonic maps from a two-torus into a complex Grassmann manifold, Int. J. Math. 6 (1995), 447-459.

Department of Mathematics, University of York, Heslington, York YO10 $5 \mathrm{DD}, \mathrm{UK}$

E-mail address: im7@york.ac.uk 